\title{
Evaluación del sistema erosivo fluvial en el volcán Popocatépetl (México) mediante análisis morfométricos
}

\author{
Miguel Castillo, ${ }^{1, *}$, Esperanza Muñoz-Salinas ${ }^{1}$, José Luis Arce ${ }^{1}$ \\ ${ }^{1}$ Instituto de Geología, Universidad Nacional Autónoma de México, Ciudad Universitaria, C.P. 04510, México D.F. \\ *castillom@geologia.unam.mx
}

\begin{abstract}
Resumen
El sistema fluvial es sensible a los cambios tectónicos, volcánicos, climáticos y litológicos, mismos que afectan las tasas de incisión e inducen cambios en la topografía del lecho de los ríos así como en laderas del sistema montañoso. El análisis de los perfiles longitudinales de ríos y la morfometría de cuencas son, por lo tanto, herramientas útiles para detectar la propagación de señales tectónicas y climáticas además de que resaltan las zonas litológicas que presentan una distinta resistencia frente a los procesos erosivos fluviales. Gran parte de los estudios sobre la erosión y evolución del relieve en los sistemas montañosos se han centrado en relieves controlados por una tectónica activa, sin embargo, los estratovolcanes han recibido menos atención, no obstante que estos llegan a construir una parte importante del relieve montañoso y más aún en la parte central de México. En esta investigación se analizan los perfiles longitudinales de los ríos principales del volcán Popocatépetl $(n=12)$ así como la morfometría de sus cuencas $(n=11)$. El objetivo es obtener información cuantitativa del relieve y explorar la relación que éste guarda con los procesos erosivos fluviales. Los resultados del análisis de los perfiles longitudinales de los ríos indican que los valores del índice normalizado de la verticalidad del canal $\left(k_{\mathrm{sn}}\right)$ así como la potencia fluvial $(A S)$ aumentan en la transición del sistema montañoso al piedemonte. Las tasas de erosión son particularmente altas aguas debajo de las cabeceras fluviales. Se detectaron knickpoints $(n=19)$, los cuales se asocian, por lo general, a la presencia de flujos de lava. Se encontró una mediana correlación $\left(\mathrm{R}^{2}=0.50 ; n=11\right)$ entre la distancia de retroceso del knickpoint y el área de drenaje y una baja a moderada correlación $\left(\mathrm{R}^{2}=0.38\right)$ entre las tasas de retroceso y el área de descarga. Los resultados obtenidos sugieren que la edad de las lavas y su dureza (competencia a la erosión) controlan las tasas de propagación de los knickpoints, los cuales retroceden a una media de $0.05 \pm 0.2 \mathrm{~m} \mathrm{a}^{-1}$. La morfometría de las cuencas revela que no existe una relación directa entre la incisión fluvial y la morfología de las laderas, a pesar de que la primera es alta en la mayoría de las barrancas. Se concluyó que el sistema fluvial se encuentra en desequilibrio, probablemente debido a la intensa actividad volcánica que ha predominado en el Popocatépetl.
\end{abstract}

Palabras clave: perfiles longitudinales de ríos, morfometría de cuencas, knickpoints, volcán Popocatépetl

\begin{abstract}
Fluvial systems are sensitive to changes in tectonics, volcanic processes, climate and lithology. These factors can modify the erosion rates producing changes in the topography of mountains, channels and hillslopes. The analysis of the longitudinal profile of rivers and the morphometry of river basins are powerful tools that allow the detection of both tectonic and climatic signals and also allow the detection of zones presenting lithologies with a high degree of resistance to erosion. Most of the studies in landscape evolution have been focused on mountain settings driven by active tectonics. The study of stratovolcanoes has, however, received less attention although they are an important part of mountainous reliefs, specially in central Mexico. Here we analyze the stream long profiles of the Popocatépetl volcano $(n=12)$ and the morphometry of its river basins $(n=11)$ with the aim to obtain the quantitative data of relief and explore the relation between the mountain topography and the erosion due to fluvial processes. Our results indicate that both the normalized channel steepness index $\left(k_{\mathrm{sn}}\right)$ and stream power $(A S)$ increase at the transition between the mountain area and piedmont. The erosion rates are particularly high downstream of headwaters. We detected the presence of knickpoints $(n=19)$, which in most of the
\end{abstract}


cases, are generated at the front of lava flows. We found a moderate correlation $\left(\mathrm{R}^{2}=0.51 ; n=11\right)$ between the distance of knickpoint retreat and the drainage area and a weak to moderate correlation $\left(\mathrm{R}^{2}=0.38\right)$ between the retreat rate and the drainage area. Our results suggest that the age and resistance of lavas to erosion control the rates of knickpoint recession which has a mean of $0.05 \pm 0.02$ $m y r^{1}$. The river basin morphometry indicates that the hillslope morphology is not tightly related to fluvial incision. Nevertheless, the channel incision rates are high in most of valleys of the study area. We conclude that the fluvial system is in disequilibrium, probably due to the continuous volcanic activity of Popocatépetl volcano.

Keywords: longitudinal profile of rivers, river basin morphometry, knickpoints, Popocatépetl volcano.

\section{Introducción}

La evolución de los sistemas montañosos, independientemente de su génesis, es controlada en gran medida por las tasas de erosión y la capacidad de transporte de sedimentos que tenga el sistema fluvial. A su vez, la incisión de los ríos controla, en muchos de los casos, la evolución y morfología de las laderas (Roering et al., 2001; Reinhardt et al., 2007; Hurst et al., 2012) y puede ser un factor para la desestabilización de las mismas (Roering et al., 2007). Diversos estudios indican que los ríos son elementos del relieve sensibles al incremento en las tasas de levantamiento tectónico, proceso que resulta en un aumento del gradiente topográfico fluvial (e.g., Kirby y Whipple, 2001; Snyder et al., 2003; Duvall et al., 2004) así como de la propagación de formas transitorias conocidas como knickpoints (Castillo y Lugo-Hubp, 2011), mismas que transmiten al relieve la caída del nivel de base por el levantamiento de un volumen de roca determinado ( $c f$. Bishop, 2007).

Las señales climáticas también se transmiten al sistema fluvial y se pueden detectar a través del análisis de la concavidad del perfil longitudinal de un río (e.g., Roe et al., 2002; Schlunegger et al., 2011; Trauerstein et al., 2013). Por ejemplo, en relieves montañosos donde existió una erosión glacial, las zonas cercanas a la cabecera de los ríos presentan una menor concavidad con respecto a los ríos que no experimentaron una glaciación (e.g., Whipple et al., 1999; Brocklehurst y Whipple, 2006), esto se explica por una intensa erosión en las partes más altas del sistema montañoso que produce una disminución del relieve local (Whipple et al., 1999). En algunos casos, los glaciares de montaña pueden producir una zona de gran concavidad más lejana de la zona de cabeceras y que corresponde a puntos donde la velocidad y la acumulación de la masa glaciar se incrementa de forma notable (Anderson et al., 2006; Norton et al., 2010). Otros factores relacionados con el clima, como es la precipitación, pueden afectar la topografía y morfología de los ríos. En sitios donde existen intensas precipitaciones, las tasas de erosión suelen incrementarse y producir una mayor concavidad en el perfil longitudinal (Schlunegger et al., 2011).

Además de los factores tectónicos y climáticos, la litología puede producir cambios importantes en la topografía de los ríos debido a la distinta resistencia que tienen los materiales frente a los procesos de incisión (e.g., Hack, 1973; Gardner, 1983; Miller, 1991; Larue, 2008). Las rocas más resistentes suelen tener pendientes más abruptas mientras que aquellas menos competentes tienen un gradiente topográfico mucho más suavizado. Hack (1973) propuso el uso de un índice de verticalidad del gradiente de los canales con el cual pueden advertir los cambios litológicos y la presencia de forzamientos tectónicos. Por lo tanto, a partir del análisis de la topografía del curso de un río, puede obtenerse información sobre las señales tectónicas y/o climáticas (Brozović et al., 1997; Brocklehurst y Whipple, 2006; Wobus et al., 2006; Whittaker et al., 2008) así como la resistencia que presenta la litología ante la erosión fluvial.

En el relieve montañoso los ríos inciden en el sustrato $\mathrm{y}$, como consecuencia, la geometría hidráulica varía con respecto a los ríos de tipo aluvial (e.g., Montgomery y Gran, 2001; Wohl, 2004; Finnegan et al., 2005; Turowski et al., 2008) así como los mecanismos que generan la erosión del lecho (Whipple, 2004). Por lo tanto, los ríos en lecho rocoso (Castillo y Lugo-Hubp, 2011) o ríos en roca (Garzón et al., 2008) son el objeto de los estudios de la evolución del relieve, no obstante que en muchos de los casos, el límite de estos ríos con respecto a los aluviales, donde predomina la movilización y degradación de sedimentos, es difícil de establecer (Turowski et al., 2008). La principal diferencia entre los ríos aluviales con respecto aquellos que se encuentran en lecho rocoso radica en los procesos fluviales que operan en el lecho. En los ríos de tipo aluvial los cambios en la morfología y erosión están limitados por la capacidad de transporte (Willgoose et al., 1994), mientras que en los ríos que inciden en el lecho están limitados por la tasa de desprendimiento de fragmentos del mismo (Howard, 1994).

No obstante que existen dos tipos generales de río, es decir, ríos en lecho rocoso y ríos aluviales, se ha observado que en la mayoría de los sistemas fluviales existe una marcada relación entre la pendiente de los canales con respecto al área de drenaje (e.g., Hack, 1957; Flint, 1974; Tarboton et al., 1991), esta última sustituye la descarga fluvial $(Q)$, de tal forma que conforme aumenta el área de drenaje disminuye la pendiente y se modela partir de la ecuación siguiente:

$$
S=k_{\mathrm{s}} A^{-\theta}
$$


donde $S$ es el gradiente del canal, $k s$ hace referencia al índice de verticalidad del canal, $A$ corresponde al área de drenaje y $\theta$ es la concavidad del canal. La Ecuación 1 es válida hasta $\sim 10^{5} \mathrm{~m}^{2}$ de área de drenaje ya que existe un área crítica $(A c$ $<10^{5} \mathrm{~m}^{2}$ ) donde predominan los procesos de ladera sobre los fluviales (Montgomery y Foufoula-Georgiou, 1993). El área crítica puede variar en función de las condiciones climáticas, de tal forma que ésta puede ser mayor en zonas áridas o disminuir en climas más lluviosos (Montgomery y Dietrich, 1988). Por otro lado, los valores de $\theta$ se encuentran en un estrecho rango de entre $\sim 0.30 \mathrm{a} \sim 0.60$ (Whipple y Tucker, 1999), sin embargo, estos pueden aumentar hasta el orden de la unidad debido a la propagación de señales tectónicas o a la presencia de forzamientos climáticos (Schoenbohm et al., 2004; Trauerstein et al., 2013).

La Ecuación 1 modela una condición donde las pendientes de los canales se encuentran niveladas al sistema erosivo, es decir, que el sistema fluvial se encuentra en equilibrio dinámico (Whipple y Tucker, 1999). Cuando existen cambios debidos a un levantamiento tectónico, forzamiento climático o a la litología, la relación entre las pendientes y área de drenaje cambia, lo cual puede incrementar el valor de $k s$ o producirse una ruptura en el escalamiento de la Ecuación 1, lo que corresponde normalmente con la presencia de knickpoints (e.g., Snyder et al., 2003; Schoenbohm et al., 2004; Whipple, 2004; Bishop et al., 2005; Wobus et al., 2006; Castillo et al., 2013; Trauerstein et al., 2013). También se recomienda tener cautela en el uso de la Ecuación 1 ya que $k s$ guarda una correlación con $\theta$, por lo tanto, es recomendable normalizar el índice de verticalidad para romper la covarianza y facilitar la comparación entre los distintos ríos de un sistema montañoso determinado (Sklar y Dietrich, 1998; Kirby et al., 2003; Wobus et al., 2006).

En distintos ambientes se ha observado que el índice normalizado de la verticalidad de los canales o valor $k_{\mathrm{sn}}$ (Wobus et al., 2006) guarda una buena correlación con las tasas de levantamiento tectónico (e.g., Cyr et al., 2010) y con la erosión (e.g., Safran et al., 2005; DiBiase et al., 2010; Kirby y Whipple, 2012), esta última se relaciona con el relieve local (Ahnert, 1970; Montgomery y Brandon, 2002; Safran et al., 2005) y con las pendientes medias de las cuencas (Binnie et al., 2007; Ouimet et al., 2009), por lo tanto los valores $k_{\mathrm{sn}}$ pueden utilizarse como indicadores robustos de procesos tectónicos y erosivos a nivel del lecho y cuenca. En los relieves volcánicos la evolución magmática puede producir numerosos flujos de lava sobre una topografía con fuertes desniveles, en tales casos lo valores $k_{\mathrm{sn}}$ podrían ser indicativos de la resistencia de los materiales volcánicos frente a la erosión o resaltar zonas de fuertes contrastes a consecuencia de la acumulación de los materiales volcánicos.

Otro enfoque alternativo al uso de la Ecuación 1 consiste en linealizar los perfiles longitudinales con una concavidad determinada e integrar el área de drenaje para producir las coordenadas $\chi$ o el denominado gráfico tipo Chi (Perron y
Royden, 2013; Royden y Perron, 2013). En el gráfico Chi el ajuste de la topografía a los procesos erosivos está dado por la linealidad entre la distancia $\chi$ y la elevación observada en el canal, de tal forma que los forzamientos tectónicos se detectan por un incremento en la verticalidad de la línea en el gráfico Chi o por rupturas en la propia linealidad (Perron y Royden, 2013).

El análisis de los perfiles longitudinales de los ríos ha demostrado ser una herramienta útil en los estudios de la evolución del relieve (Wobus et al., 2006) aunque su uso se ha centrado más en demostrar la presencia del equilibrio dinámico (e.g., Montgomery, 2001; Stolar et al., 2007; Cyr et al., 2010), detectar zonas sujetas a forzamientos tectónicos o a evaluar la propagación de knickpoints a consecuencia del incremento en las tasas de levantamiento (Duvall et al., 2004; Harkins et al., 2007; Attal et al., 2008; Finnegan et al., 2008; Attal et al., 2011). La evolución del relieve en ambientes volcánicos ha recibido menos atención aunque hay algunas excepciones (e.g., Seidl et al., 1994; Stock y Montgomery, 1999; Whipple et al., 2000; Ye et al., 2013).

La importancia de entender la evolución de relieves volcánicos radica en que estos producen grandes desniveles, como es el caso de los estratovolcanes, donde se presupone que las tasas de erosión pueden ser altas debido al incremento del gradiente topográfico y donde pueden existir contrastes en dichas tasas debido al aporte de distintos materiales por parte de la actividad volcánica. En el caso donde la actividad volcánica ha cesado, los estratovolcanes son erosionados casi en su totalidad y queda solamente el conducto principal (Ollier, 1988). Sin embargo, en los estratovolcanes activos se pueden generar distintas respuestas por parte del sistema erosivo. En los casos donde predomina la actividad efusiva las tasas de erosión pueden ser bajas o ser nulas, en otros casos, en el periodo post-eruptivo se puede dar una rápida erosión de los materiales piroclásticos (Thouret, 1999). Debido a que los estratovolcanes activos son superficies que experimentan cambios drásticos en tiempos cortos, estos pueden ser relieves en transitoriedad ante los procesos erosivos fluviales. De acuerdo con lo anterior, resalta la pregunta de si los ríos en volcanes activos tienen un perfil cóncavo, como lo indica la Ecuación 1 o estos se encuentran interrumpidos por knickpoints que resultan de los flujos de lava o de depósitos volcánicos consolidados, entonces si hay knickpoints ¿cuál es su tasa de retroceso?, ¿existe una relación entre la incisión y la morfología de las laderas? Motivados por la necesidad de explorar la relación entre los procesos erosivos y la topografía de un estratovolcán activo decidimos estudiar los perfiles longitudinales y la morfometría de las principales cuencas del volcán Popocatépetl.

El Popocatépetl es una estructura que presenta un desnivel de $\sim 3000 \mathrm{~m}$, condición que sugiere a priori la presencia de altas tasas de erosión. Sin embargo, los estudios sobre la erosión fluvial son escasos, aunque es bien conocida la alta dinámica erosivo-acumulativa asociada a la generación de lahares (Robin y Boudal, 1987; Capra 
et al., 2004; Espinasa-Pereña y Martín-Del Pozzo, 2006; Muñoz-Salinas et al., 2007). En este estudio se provee de información cuantitativa sobre la topografía de los lechos y las cuencas, se reporta la localización de knickpoints formados por flujos de lava de distintas edades y sus tasas de retroceso, de lo cual se advierte la predominancia de una alta erosión fluvial en la mayoría de las barrancas que se encuentran en el volcán.

\section{2. Área de estudio}

El Popocatépetl es un estratovolcán activo de $\sim 5400$ $\mathrm{m}$ de altitud, con un desnivel de $\sim 3000 \mathrm{~m}$ desde su base (Macías, 2005). El volcán se encuentra situado en la porción central del Cinturón Volcánico Trans-Mexicano, en el extremo sur de la Sierra Nevada (Palacios, 1996) (Figura 1). El Popocatépetl está $70 \mathrm{~km}$ al SE de la Ciudad de México y a $\sim 40 \mathrm{~km}$ al $\mathrm{O}$ de la zona metropolitana de Puebla donde habitan $\sim 20000000$ y 2000000 de personas respectivamente (INEGI, 2012). No obstante el interés científico y el peligro geológico que representa el volcán, la información sobre la geología y cronología de los distintos materiales sólo están disponibles de forma fragmentada (Schaaf et al., 2005). La compilación más reciente sobre los productos volcánicos y las edades es la propuesta por Espinasa-Pereña y Martín-Del Pozzo (2006) quienes identifican una serie de unidades morfoestratigráficas en el volcán.

La parte más antigua del edificio se compone de lavas andesíticas y dacíticas del volcán Nexpayantla o volcán primitivo (Robin y Boudal, 1987) y corresponden a la primera fase constructiva del Popocatépetl (EspinasaPereña y Martín-Del Pozzo, 2006). Se estima que la edad de los materiales más antiguos es de $\sim 730000$ años y corresponden a lavas que están dentro del cron de Brunhes (Conte et al., 2004). La barranca de Nexpayantla es uno de los vestigios del antiguo edificio, misma que sobresale por su gran desnivel (río 1; Figura 1). El edificio primitivo se destruyó $\sim 200000$ años y formó una caldera sobre la cual se construyó el nuevo edificio conocido como El Fraile (Robin y Boudal, 1987), el cual está compuesto por derrames andesíticos y dacíticos (Macías, 2005). Entre 50000 y más de 30000 años, ocurrió la destrucción del Fraile por un evento de tipo Bezymianny el cual produjo un depósito masivo de avalancha de escombros localizado al sur del volcán, así como flujos piroclásticos de ceniza y pómez (Robin y Boudal, 1987). Posterior a la destrucción del Fraile se inició la construcción del cono moderno del Popocatépetl (Macías, 2005).

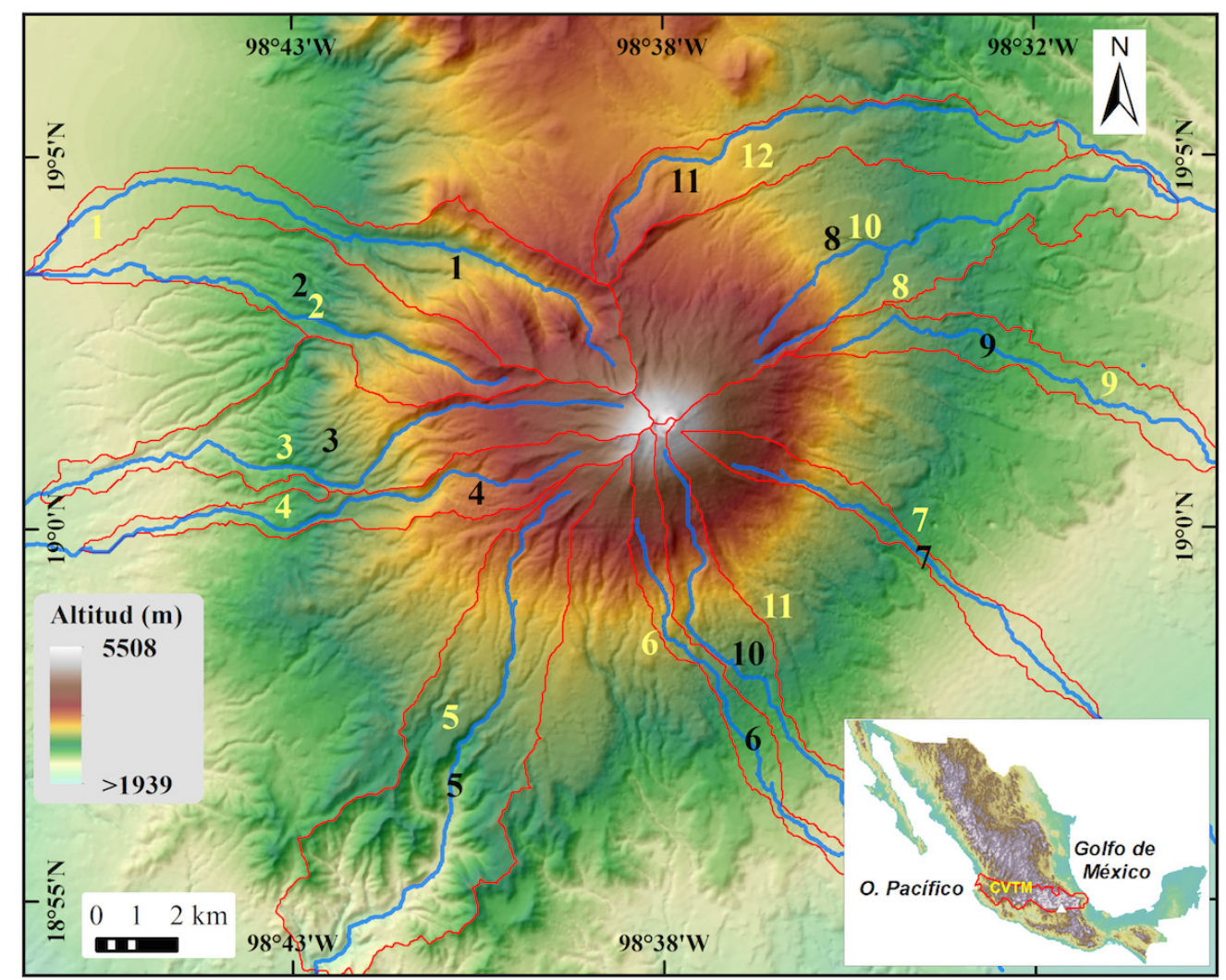

Figura 1. Localización del volcán Popocatépetl. El mapa del recuadro indica la posición del volcán Popocatépetl dentro del Cinturón Volcánico TransMexicano. Las líneas azules indican los ríos estudiados, en rojo se marcan las cuencas, los números en negro corresponden con el identificador de la cuenca (consultar la Tabla 3) y los números en color amarillo claro corresponden con el identificador asignado a cada río (para los nombres consultar la Tabla 1). 
Hace $\sim 23000$ años en el Popocatépetl tuvo lugar un evento tipo Santa Elena el cual consistió de un colapso de la parte sur del edificio volcánico, dando lugar al emplazamiento de depósitos de avalancha de escombros, seguido de la emisión de depósitos piroclásticos (depósitos de pómez de caída) los cuales se encuentran sobre el sector sur del volcán (Siebe et al., 1995). En los últimos 20000 años la actividad volcánica en el Popocatépetl ha estado asociada a varios eventos de tipo pliniano los cuales han producido depósitos de caída de pómez y flujos de ceniza (Macías, 2005; Schaaf et al., 2005). Los depósitos piroclásticos alternan con derrames de lava de composición andesítica a dacítica (Macías, 2005). Hoy en día predomina la formación de domos de lava en el interior del cráter y su subsecuente destrucción produce flujos piroclásticos (Franco, 2005). De acuerdo a las unidades morfoestratigráficas de EspinasaPereña y Martín-Del Pozzo (2006) los derrames de lava más antiguos ( $>23000$ años) se encuentran en el flanco noroccidental del edificio. En el flanco sur se encuentran los derrames de lava de $\sim 23000$ a $\sim 14000$ años y los derrames más recientes se localizan en el flanco nororiental del volcán con una edad aproximada de 13000 a 900 años.

Hasta ahora no existen datos suficientes sobre las tasas de erosión por procesos fluviales en el volcán (Franco, 2009), no obstante, la presencia de los glaciares y sus posibles efectos en la topografía fueron reconocidos de forma más o menos temprana (White, 1981). La cronología y la reconstrucción de los procesos glaciales en el Popocatépetl se ha visto impedida por la actividad volcánica, la cual ha sepultado gran parte de los depósitos y formas asociados a estos (Lachniet y Vazquez-Selem, 2005). Asimismo, la cronología de las glaciaciones del centro de México no está del todo formalizada, ya que los avances glaciales están basados en datos cualitativos con un incompleto registro de todos los volcanes (Vázquez-Selem y Heine, 2011) y el nombre en algunos de los mismos avances glaciales varían de acuerdo con los volcanes estudiados (cf. Heine, 1994). El registro más completo de los avances glaciales del centro de México son los presentados por Vázquez-Selem y Heine (2011) para el volcán Iztaccíhuatl e incluyen edades $\operatorname{con}^{36} \mathrm{Cl}$ cosmogénico. Estos mismos autores proponen utilizar los datos del Iztaccíhualt como indicador de las glaciaciones del Wisconsoniano tardío hasta el Holoceno para el centro de México. La cercanía geográfica y la diferencia de $\sim 45 \mathrm{~m}$ de la Línea de Equilibrio Altitudinal (LEA) actual entre el Popocatépetl e Iztaccíhuatl (White, 2002) son condiciones que permiten correlacionar de forma confiable los avances glaciales del Iztaccíhuatl con los del Popocatépetl.

Las edades de ${ }^{36} \mathrm{Cl}$ indican que los principales avances ocurrieron hace $\sim 205000,21000,17000,12000,8000$, $<1000$ años (Vázquez-Selem y Heine, 2011). El avance más antiguo erosionó las lavas del volcán Nexpayantla (Espinasa-Pereña y Martín-Del Pozzo, 2006) los avances de 17000 hasta 8000 años los identifican Espinasa-Pereña y Martín-Del Pozzo (2006) hacia el sector occidental del volcán, sin embargo, estos autores indican que la presencia de morrenas no fue verificada en campo y su localización es tentativa. Las morrenas asociadas a los avances de $<1000$ años se encuentran en los flancos sur, occidental y norte de la superficie cumbral (Espinasa-Pereña y Martín-Del Pozzo, 2006). La importancia de la presencia de los glaciares en ambientes de montaña no sólo radica en su capacidad como agentes de erosión y producción de sedimentos (Church y Ryder, 1972), sino también como agentes asociados al desencadenamiento de lahares, como ha ocurrido en el Popocatépetl (e.g., Capra et al., 2004; Julio-Miranda et al., 2005; Muñoz-Salinas et al., 2007). Hoy en día el retroceso de los glaciares en el Popocatépetl es un hecho demostrado que ha alcanzado tasas de $\sim 40 \mathrm{~m} \mathrm{a}^{-1}$ (Delgado-Granados, 1997), sin embargo, las bajas temperaturas en la zona cumbral, junto a la precipitación en forma de nieve son también condiciones propicias para la formación de lahares (Palacios et al., 2001; Muñoz-Salinas et al., 2010).

La presencia de lahares of flujos de derrubios son procesos que han predominado en la historia geológica del volcán (e.g., Siebe et al., 1995; Julio-Miranda y Delgado-Granados, 2003; Capra et al., 2004; Muñoz-Salinas et al., 2007) y constituyen una parte importante del relleno de las barrancas (Franco, 2005, 2009; Espinasa-Pereña y Martín-Del Pozzo, 2006), sin embargo no existen estudios que cuantifiquen la degradación de los sedimentos asociados a flujos de derrubios o procesos fluviales en las barrancas del volcán (Franco, 2009). En la Figura 2 se presentan las principales unidades morfoestratigráficas del volcán Popocatépetl. La datación de capas de cenizas y flujos piroclásticos en distintas partes del volcán permite evaluar, grosso modo, las tasas de erosión en los ríos. Franco (2009) reporta tasas de erosión en el flanco norte del volcán de 0.0009 a $0.2 \mathrm{~m}$ $\mathrm{a}^{-1}$ en la cabecera de los afluentes del río "Pelagallina" y de $0.008 \mathrm{a} \sim 0.01 \mathrm{~m} \mathrm{a}^{-1}$ sobre los ríos Pelagallina y La Venta respectivamente. Con base en las tasas estimadas y en una interpretación geomorfológica, Franco (2009) elabora una clasificación de la dinámica de las barrancas en el flanco norte del volcán, sin embargo, en su estudio no se proveen datos de descarga (o área de drenaje) ni de las pendientes del lecho, no obstante que estos dos factores son clave para estimar las tasas de erosión en los lechos (Howard y Kerby, 1983).

\section{Materiales y métodos}

Los perfiles longitudinales y la cuenca de los ríos se obtuvieron a partir de un modelo digital de elevación (MDE) con una resolución de $20 \mathrm{~m}$ por pixel. El MDE se extrajo de la interpolación de las curvas de las cartas topográficas Huejotzingo, Amecameca de Juárez, Cuautla y Atlixco a escala 1:50000 publicadas por el Instituto Nacional de Geografía Estadística e Informática (INEGI) mediante el uso de un Sistema de Información Geográfica (SIG). Se seleccionaron los principales ríos $(n=12)$ que inciden en el volcán (Figura 1). Los datos de elevación, área de drenaje 


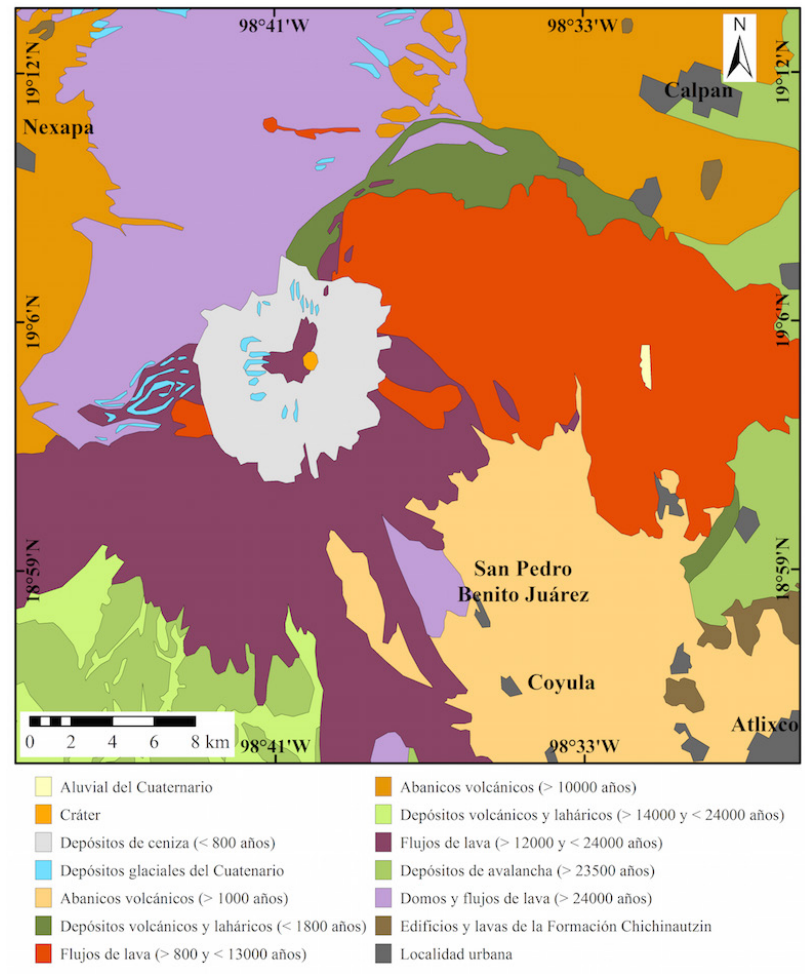

Figura 2. Mapa de las principales unidades morfoestratigráficas del volcán Popocatépetl (tomado y modificado de Espinasa-Pereña y Martín del Pozzo, 2006).

y distancia desde la divisoria se obtuvieron del MDE con el uso de la herramienta Stream Profiler, misma que está disponible en línea en http://www.geomorphtools.org y que contiene una serie de scripts para extraer la información hidrológica en el SIG. Las pendientes de canal se estimaron a partir de los datos de elevación y la distancia desde la divisoria.

Con los datos obtenidos de los perfiles longitudinales se evaluó la relación entre la pendiente y el área de drenaje con el método propuesto por Wobus et al. (2006), se utilizó una concavidad de referencia $\left(\theta_{\text {ref }}\right)$ de 0.45 en la Ecuación 1 para facilitar la comparación entre los distintos ríos que inciden en el volcán así como los cambios en los distintos tramos de un mismo río. Por la relación que existe entre valores $k_{\mathrm{sn}}$ con las tasas de erosión (Kirby y Whipple, 2012), los valores altos de $k_{\mathrm{sn}}$ indican, en la mayoría de los casos, zonas donde se presupone que existe una mayor incisión y zonas donde existe una litología resistente frente a la erosión fluvial, en cuyo caso los valores de $k_{\text {sn }}$ son bajos. Con el objeto de estimar la verticalidad de los canales se estimó el valor medio $k_{\mathrm{sn}}$ para cada uno de los ríos.

Complementario a los valores $k_{\mathrm{sn}}$ se evaluó la potencia fluvial $(\Omega)$ en cada perfil, mediante la simplificación del índice de erosión propuesto por Finlayson y Montgomery (2003), de tal forma que:

$$
\Omega=\Sigma(A) S
$$

$S$ es la pendiente del canal. La simplificación de $\Omega$ se hizo porque no existen datos sobre el exponente que resulta de la relación entre la descarga y el área de drenaje, y además las estaciones meteorológicas son pocas alrededor del volcán. El valor de la integral hipsométrica $\left(H_{i}\right)$ (Strahler, 1952) se determinó a partir de la ecuación propuesta por Pike y Wilson (1971) en la que:

$$
H_{i}=\left(\mathrm{Z}_{\text {media }}-\mathrm{Z}_{\text {min }}\right) /\left(\mathrm{Z}_{\text {max }}-\mathrm{Z}_{\text {min }}\right)
$$

donde $Z$ corresponde a la elevación del canal y las subscripciones corresponden con los valores estadísticos de la media, mínima y máxima. Con los datos de distancia y rango de elevación se determinó el gradiente total de cada uno de los ríos. Complementario al análisis de la pendiente y área de descarga, se hizo la linearización de los perfiles con base en el método sugerido por Perron y Royden (2013), de los cuales se extrajo el exponente $(\mathrm{m} / \mathrm{n})$ el cuál se comparó con los datos morfométricos descritos. La métrica de los canales se presenta en la Tabla 1.

Para cada uno de los ríos se extrajeron los principales knickpoints mediante la interpretación de los gráficos del gradiente del canal versus el área de drenaje, con ayuda de la herramienta geomorphotools procesada en el paquete MATLAB ${ }^{\circledR}$. Los knickpoints fueron exportados al SIG donde se utilizó un MDE proveniente de datos LIDAR con una resolución de $5 \mathrm{~m}$ por pixel y disponible en la página web del INEGI (para confirmar la localización de las formas mencionadas). Debido a que los knickpoints resultan de diversos factores genéticos (Castillo y Lugo-Hubp, 2011), en esta investigación se estimó el caso de los knickpoints que retroceden a consecuencia de cambios bruscos en la topografía del río, como resultado de los flujos de lava aunque no están descartados la formación de knickpoints en otro tipo de depósitos volcánicos en el Popocatépetl. Con el uso de un modelo sombreado derivado de MDE de $5 \mathrm{~m}$ se midió la distancia del knickpoint $(n=11)$ hacia el frente de lava donde se supone que se inició el retroceso. La edad de las lavas y las tasas de retroceso se establecieron con las edades publicadas por Espinasa-Pereña y Martín del Pozzo (2006), en su tabla morfoestratigráfica. Los datos de los knickpoints se presentan en la Tabla 2.

Las principales cuencas del volcán Popocatépetl se extrajeron del mapa de acumulación de flujos derivado del MDE de $20 \mathrm{~m}$ de resolución (Figura 1). Para cada cuenca se extrajo el valor medio del índice normalizado del canal, la pendiente media así como los valores de convexidad y de concavidad. Para determinar el valor medio de la concavidad y convexidad se clasificó el mapa de curvatura, extraído del MDE, de tal forma que los valores menores a -0.25 y mayores a 0.25 corresponden a superficies cóncavas y convexas respectivamente. Como los valores cercanos a 0 en el mapa de curvatura corresponden a superficies llanas, la clasificación utilizada aquí permitió distinguir las divisorias y el fondo de los valles. Los datos de las cuencas extraídas se encuentran resumidos en la Tabla 3.

donde $A$ es el área de drenaje, misma que sustituye a $Q$ y 
Tabla 1. Datos morfométricos de los ríos principales que inciden sobre el volcán Popocatépetl.

\begin{tabular}{|c|c|c|c|c|c|c|c|c|c|}
\hline ID & Nombre & $\operatorname{UTM}(X)^{a}$ & $\begin{array}{l}\text { UTM } \\
(\mathbf{Y})^{\mathbf{a}}\end{array}$ & $\begin{array}{c}\text { Área de } \\
\text { drenaje }\left(\mathbf{k m}^{2}\right)\end{array}$ & $\begin{array}{l}\text { Desnivel } \\
\text { (m) }\end{array}$ & $\begin{array}{l}\text { Valor medio de } k_{\mathrm{sn}} \\
\qquad\left(\mathrm{m}^{0.9}\right)^{\mathrm{b}}\end{array}$ & $\begin{array}{c}\text { Integral } \\
\text { hipsométrica }\end{array}$ & $\begin{array}{l}\text { Gradiente } \\
\quad(\mathbf{m} / \mathbf{m})\end{array}$ & $\begin{array}{l}\text { Índice de concavidad } \\
\qquad(\mathrm{m} / \mathrm{n})^{\mathrm{c}}\end{array}$ \\
\hline 1 & Nexpayantla & 538546 & 2104316 & 50.9 & 2388 & $124 \pm 42$ & 0.26 & 8.1 & 0.50 \\
\hline 2 & Huayatlaco & 536386 & 2104536 & 50.9 & 1760 & $101 \pm 42$ & 0.31 & 8.5 & 0.42 \\
\hline 3 & Yancuecole & 539006 & 2103696 & 32.6 & 2917 & $142 \pm 65$ & 0.32 & 6.3 & 0.33 \\
\hline 4 & Huitlastoc & 538106 & 2102916 & 30.3 & 2440 & $132 \pm 48$ & 0.34 & 7.1 & 0.37 \\
\hline 5 & Hueyetlaquixtle & 537866 & 2102216 & 38.4 & 2329 & $145 \pm 68$ & 0.36 & 7.4 & 0.23 \\
\hline 6 & Hueyetlaco & 538806 & 2102176 & 29.1 & 2661 & $123 \pm 31$ & 0.33 & 6.9 & 0.43 \\
\hline 7 & Xalteluco & 540366 & 2102896 & 83.6 & 2926 & $106 \pm 46$ & 0.25 & 7.2 & 0.56 \\
\hline 8 & Xalilpilcayatl & 540766 & 2104176 & 101.6 & 2200 & $115 \pm 64$ & 0.27 & 7.9 & 0.36 \\
\hline 9 & Teletra & 542986 & 2105296 & 10.9 & 1444 & $75 \pm 73$ & 0.33 & 9.3 & 0.47 \\
\hline 10 & Cuacuyulula & 540526 & 2104196 & 101.6 & 2331 & $123 \pm 71$ & 0.26 & 7.6 & 0.34 \\
\hline 11 & Comuatlatenco & 539346 & 2103456 & 29.1 & 3268 & $142 \pm 32$ & 0.31 & 5.9 & 0.50 \\
\hline 12 & Barranca Seca & 538066 & 2107056 & 101.6 & 1596 & $95 \pm 42$ & 0.37 & 13.6 & 0.25 \\
\hline
\end{tabular}

${ }^{a}$ Coordenadas estimadas a partir del datum WGS84. Coordenadas desde la cabecera del río.

${ }^{\mathrm{b}}$ Valores normalizados $\operatorname{con} \theta_{\text {ref }}=0.45$.

${ }^{\text {c } C o n c a v i d a d ~ e s t i m a d a ~ p o r ~ e l ~ m a ́ x i m o ~ v a l o r ~ d e ~ c o r r e l a c i o ́ n ~(P e r r o n ~ y ~ R o y d e n, ~ 2013) . ~}$

Tabla 2. Datos de los knickpoints del volcán Popocatépetl

\begin{tabular}{|c|c|c|c|c|c|c|c|c|c|}
\hline ID & UTM $(X)^{\mathbf{a}}$ & $\operatorname{UTM}(Y)^{a}$ & $\begin{array}{c}\text { Área de drenaje } \\
\text { en el knickpoint } \\
\qquad\left(\mathbf{k m}^{2}\right)\end{array}$ & $\begin{array}{l}\text { Altitud } \\
\text { (m) }\end{array}$ & $\begin{array}{l}\text { Distancia a la } \\
\text { divisoria (m) }\end{array}$ & $\begin{array}{l}\text { Distancia de } \\
\text { retroceso } \\
(\mathbf{m})^{b}\end{array}$ & $\begin{array}{l}\text { Área de drenaje el el } \\
\text { punto inicial del } \\
\text { retroceso }\left(\mathbf{k m}^{2}\right)\end{array}$ & Edad (años) ${ }^{c}$ & $\begin{array}{c}\text { Tasa de } \\
\text { retroceso } \\
\left(\mathrm{m} \mathrm{a}^{-1}\right)\end{array}$ \\
\hline 1 & 538086 & 2105096 & 0.4 & 4185 & 983 & - & - & - & - \\
\hline 2 & 537586 & 2105596 & 1.5 & 3866 & 1772 & - & - & - & - \\
\hline 3 & 535846 & 2107156 & 7.2 & 3368 & 4610 & 1810 & - & - & - \\
\hline 4 & 531686 & 2105656 & 5.8 & 2995 & 5389 & - & - & - & - \\
\hline 5 & 533646 & 2103676 & 2.9 & 3601 & 5692 & - & - & - & - \\
\hline 6 & 531126 & 2101976 & 10.1 & 3003 & 9223 & 512 & 10.2 & 23655 & 0.02 \\
\hline 7 & 533926 & 2102036 & 3.8 & 3480 & 4911 & 526 & 4.5 & 23655 & 0.02 \\
\hline 8 & 530246 & 2100836 & 8.2 & 2857 & 9365 & 1094 & 8.7 & $14430 \pm 190$ & 0.08 \\
\hline 9 & 535966 & 2100536 & 1.4 & 3748 & 1817 & - & - & - & - \\
\hline 10 & 535726 & 2098296 & 3.5 & 3360 & 4351 & 933 & 5.8 & 23655 & 0.04 \\
\hline 11 & 534326 & 2095056 & 11.1 & 2741 & 9530 & 1245 & 13.4 & 23655 & 0.05 \\
\hline 12 & 539086 & 2099916 & 1.3 & 3746 & 2098 & 304 & 1.5 & 14430 & 0.02 \\
\hline 13 & 542846 & 2102016 & 1.8 & 3555 & 2862 & 652 & 2.1 & $14430 \pm 190$ & 0.05 \\
\hline 14 & 543066 & 2105696 & 1 & 3759 & 2983 & - & - & - & - \\
\hline 15 & 545566 & 2107916 & 9.2 & 2989 & 6793 & 1107 & 10.6 & $12900 \pm 40$ & 0.09 \\
\hline 16 & 550566 & 2109676 & 38 & 2604 & 13070 & 274 & 38.1 & $1825 \pm 175$ & 0.15 \\
\hline 17 & 543026 & 2106856 & 1.4 & 3614 & 3903 & 667 & 2.1 & $12900 \pm 40$ & 0.05 \\
\hline 18 & 542086 & 2110836 & 6.5 & 3292 & 7144 & 2165 & - & - & - \\
\hline 19 & 546186 & 2111276 & 12.1 & 2878 & 11799 & 919 & 12.5 & $4965 \pm 65$ & 0.19 \\
\hline
\end{tabular}

${ }^{a}$ Coordenadas estimadas a partir del datum WGS84.

${ }^{\mathrm{b}}$ Los valores faltantes (-) corresponden a knickpoints en los cuales no se observa un retroceso.

${ }^{c}$ Edades de tomadas del mapa morfoestratigráfico compiladas por Espinasa y Martín del Pozzo (2007).

\section{Resultados y discusión}

El análisis topográfico de los perfiles longitudinales indica que la mayoría de los ríos del volcán Popocatépetl tienen la morfología de un perfil cóncavo (Figura 3, ID: 1,
$6,7,10$ y 11), como ocurre en gran parte de los sistemas fluviales (e.g., Flint, 1974; Willgoose, 1994; Whipple y Tucker, 1999). Sin embargo, la concavidad se hace patente cuando el área de drenaje es $>5 \times 10^{6} \mathrm{~m}^{2}$. En áreas de drenaje $<5 \times 10^{6} \mathrm{~m}^{2}$ los ríos tienen un perfil recto, donde resalta la 
Tabla 3. Datos topográficos de las cuencas del volcán Popocatépetl.

\begin{tabular}{|c|c|c|c|c|c|c|c|}
\hline ID & $\begin{array}{c}\text { Área de drenaje } \\
\qquad\left(\mathrm{km}^{2}\right)\end{array}$ & $\operatorname{UTM}(\mathbf{X})^{\mathrm{a}}$ & $\mathbf{U T M}(\mathbf{Y})^{\mathbf{a}}$ & $\begin{array}{c}\text { Gradiente medio } \\
\text { (grados) }\end{array}$ & $\begin{array}{l}\text { Valor medio del } k_{\mathrm{sn}} \\
\qquad\left(\mathrm{m}^{0.9}\right)\end{array}$ & Concavidad media & Convexidad media \\
\hline 1 & 26.1 & 532825 & 2107340 & $21.1 \pm 14.5$ & $120 \pm 35$ & $-0.83 \pm 0.63$ & $0.79 \pm 0.51$ \\
\hline 2 & 20.6 & 530777 & 2106470 & $18.9 \pm 11.5$ & $91 \pm 34$ & $-0.96 \pm 0.67$ & $0.8 \pm 0.49$ \\
\hline 3 & 24.6 & 531931 & 2103290 & $20.3 \pm 10.3$ & $123 \pm 60$ & $-0.97 \pm 0.65$ & $0.87 \pm 0.53$ \\
\hline 4 & 11.4 & 533142 & 2101740 & $18.1 \pm 10.2$ & $130 \pm 39$ & $-0.79 \pm 0.53$ & $0.77 \pm 0.49$ \\
\hline 5 & 38.3 & 534139 & 2095080 & $22.2 \pm 12.5$ & $119 \pm 60$ & $-0.99 \pm 0.69$ & $0.93 \pm 0.63$ \\
\hline 6 & 8.0 & 540690 & 2097620 & $15.6 \pm 10.0$ & $120 \pm 33$ & $-0.74 \pm 0.54$ & $0.69 \pm 0.43$ \\
\hline 7 & 8.3 & 545387 & 2100210 & $15.6 \pm 10.7$ & $109 \pm 50$ & $-0.85 \pm 0.67$ & $0.68 \pm 0.42$ \\
\hline 8 & 41.8 & 543647 & 2107530 & $14.4 \pm 9.10$ & $118 \pm 48$ & $-0.70 \pm 0.49$ & $0.65 \pm 0.40$ \\
\hline 9 & 10.8 & 548488 & 2104740 & $10.0 \pm 7.0$ & $75 \pm 20$ & $-0.67 \pm 0.44$ & $0.65 \pm 0.39$ \\
\hline 10 & 12.3 & 541556 & 2097650 & $17.5 \pm 10.1$ & $142 \pm 33$ & $-0.80 \pm 0.55$ & $0.70 \pm 0.42$ \\
\hline 11 & 19.1 & 543266 & 2109990 & $12.3 \pm 7.6$ & $92 \pm 38$ & $-0.74 \pm 0.51$ & $0.63 \pm 0.30$ \\
\hline
\end{tabular}

${ }^{a}$ Coordenadas estimadas a partir del datum WGS84.
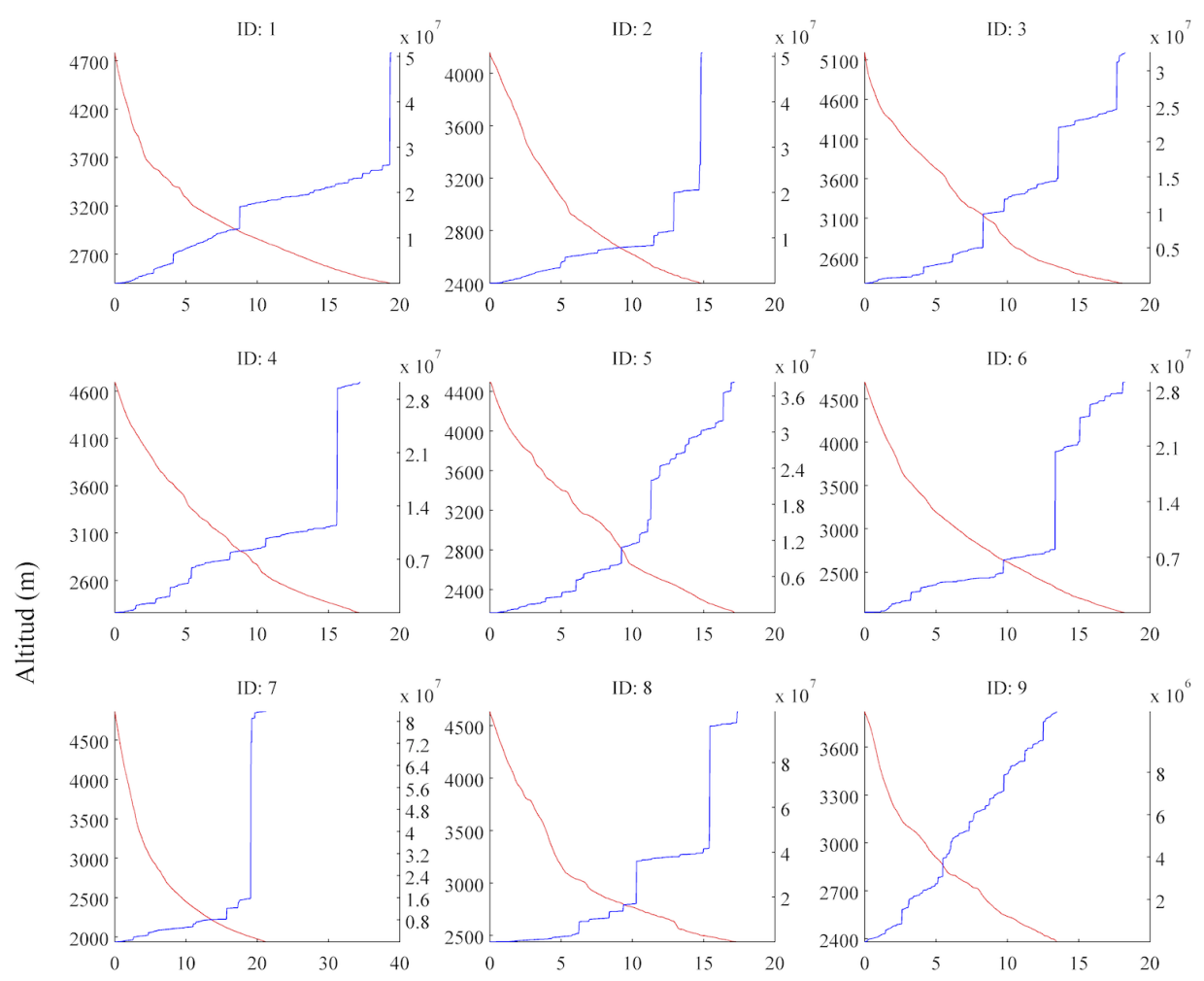

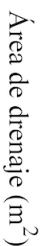

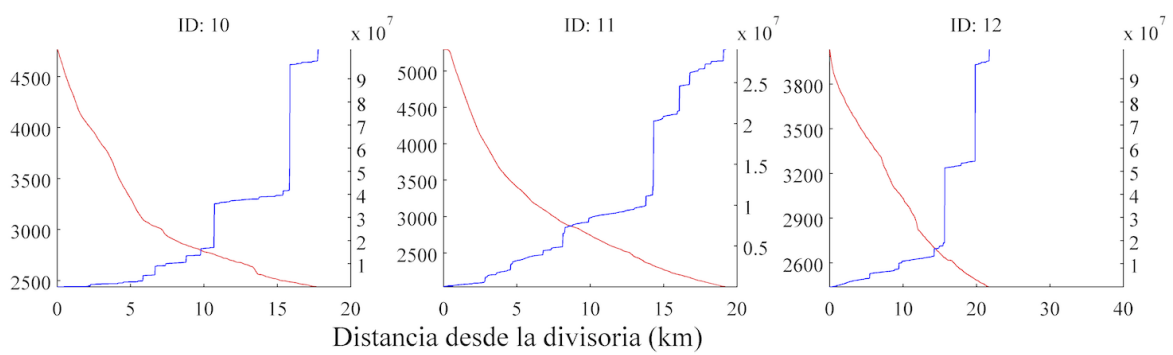

Figura 3. Perfiles longitudinales y área de drenaje del volcán Popocatépetl. Los perfiles topográficos (línea roja, eje vertical izquierdo) revelan una morfología cóncava de los perfiles a partir de área de drenaje de $5 \times 10^{6} \mathrm{~m}^{2}$ de área de drenaje (línea azul, eje vertical derecho). Nótese la presencia de knickpoints en la mayoría de los perfiles topográficos. 
presencia de knickpoints (Figura 3, ID: 3, 4, 5, 8, 9 y 12). El desarrollo de la concavidad en el perfil longitudinal a partir de un área mayor de drenaje (i.e. $>10^{5} \mathrm{~m}^{2}$ ) sugiere que los procesos de ladera, como son los flujos de derrubios, operan sobre un área más extensa con respecto a otros ambientes montañosos, donde el cambio entre los procesos fluviales con respecto a los de ladera es $10^{5} \mathrm{~m}^{2}$ de área de drenaje (Montgomery, 2001). Aunque el clima puede ser un factor importante en la localización del área crítica donde comienzan los procesos fluviales (Montgomery y Dietrich, 1988) en el caso del volcán Popocatépetl las condiciones de precipitación anual de $\sim 1700 \mathrm{~mm}$ (Fernández-Eguiarte et al., 2014) sugieren que el área crítica para el inicio de los procesos fluviales debe ser cercana a los $10^{5} \mathrm{~m}^{2}$ de área de drenaje. Es muy probable que tanto la historia eruptiva, la topografía del edificio y el grado de compactación de los materiales volcánicos sean factores que controlan el desarrollo de los flujos de derrubios, los cuales pueden alcanzar zonas lejanas a la cima, como se ha registrado en algunas barrancas (Capra et al., 2004) o desencadenarse en áreas de drenaje superiores a los $10^{5} \mathrm{~m}^{2}$. En ninguno de los perfiles longitudinales cercanos a la cabecera se observó la morfología típica del modelado glacial caracterizada por una zona de bajo gradiente topográfico con un relieve suavizado o con una superficie cóncava en la cabecera, lo cual confirma que gran parte de ese tipo de relieve ha sido sepultado por productos volcánicos jóvenes o ha sido erosionado por procesos fluviales y de laderas.

Para el caso del volcán Popocatépetl, ninguno de los ríos mostró un escalamiento nítido entre las pendientes del canal y el área de drenaje (Figura 4). En los gráficos de la Figura 4 también se observa que en la mayoría de los ríos, con
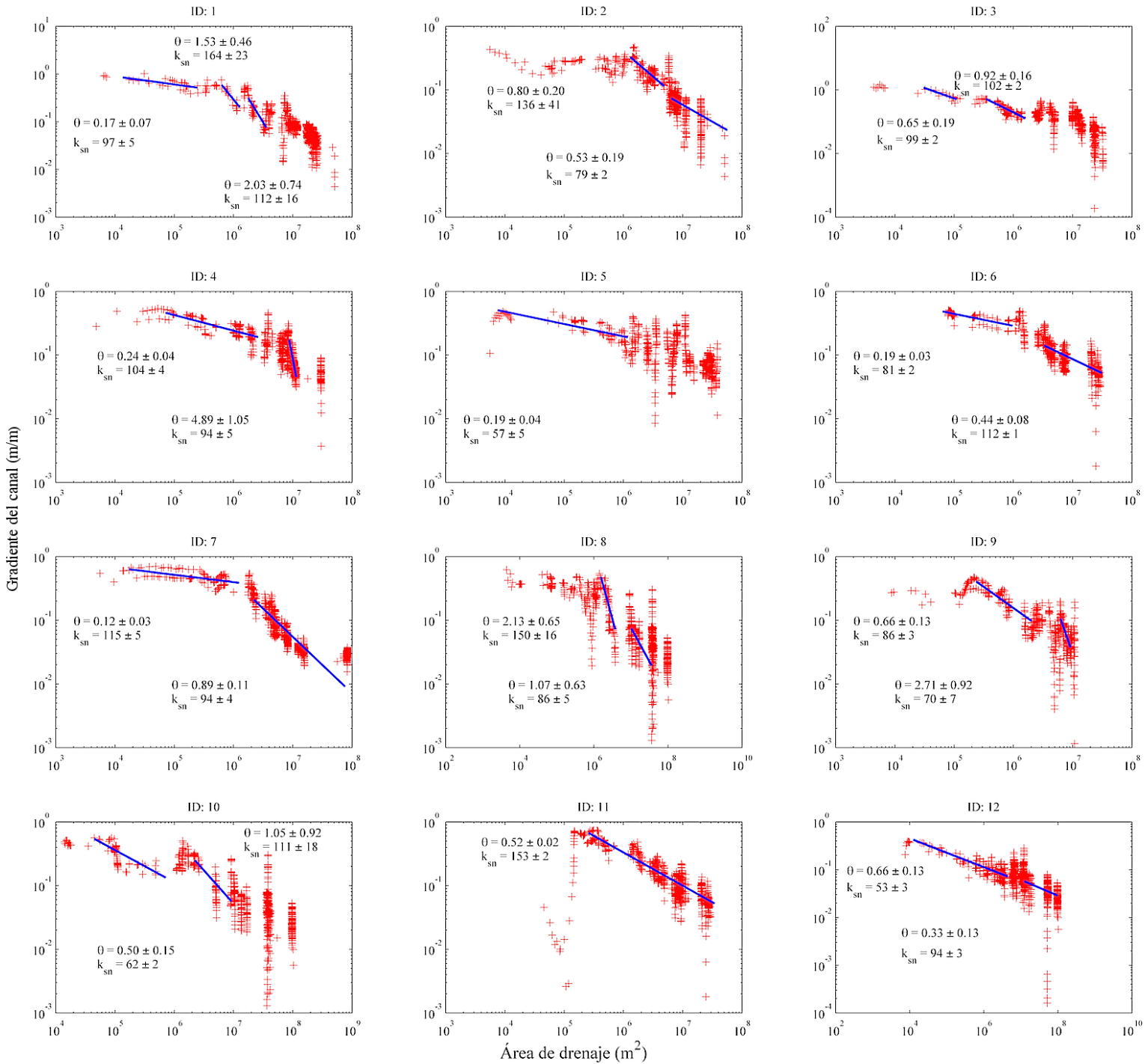

Figura 4. Gráficos del gradiente del canal versus área de drenaje. Todas las regresiones (líneas azules) son significativas ( $\mathrm{p}<0.05$ ) y con un valor de $\mathrm{R}^{2}$ $>0.60$, el valor $k_{\mathrm{sn}}$ se obtuvo con $\theta_{\text {ref }}=0.45$. Todos los ríos tienen altos valores de gradiente del canal a lo largo de su curso. Obsérvese la presencia de valores altos de $\theta$ ocurren en área de drenaje $>10^{5} \mathrm{~m}^{2}$. 
excepción de los ríos 3 y 12 (Figura 4), la relación entre el gradiente de los canales y el área de drenaje se inicia en áreas de drenaje $>10^{5} \mathrm{~m}^{2}$ y los valores de $\theta$ presentan a su vez una gran variabilidad (desviación estándar $=1.27$ ). Debido a que los valores $k_{\mathrm{sn}}$ están correlacionados con la erosión (e.g., Kirby y Whipple, 2012), en este trabajo se interpreta que las tasas de erosión en las barrancas del volcán Popocatépetl, deben ser altas en la zona de transición entre el relieve montañoso y la superficie de piedemonte (Figura 1), ya que los valores $k_{\mathrm{sn}}$ lejanos a la cabecera $\left(>10^{5} \mathrm{~m}^{2}\right.$ de área de drenaje) suelen ser altos $\left(k_{\mathrm{sn}}>100\right)$ y corresponden a zonas donde los canales tienen un mayor gradiente topográfico. En algunos sistemas montañosos se ha observado que la presencia de valores de $\theta$ altos $(\theta>0.6)$ en áreas de drenaje $>10^{5} \mathrm{~m}^{2}$ denotan altas tasas de incisión por la influencia de la precipitación, la presencia de un rápido levantamiento tectónico o la combinación de ambas (e.g., Trauerstein et al., 2013). En el caso del volcán Popocatépetl el incremento en la concavidad puede estar relacionado con el aporte de los tributarios, los cuales incrementan de forma significativa la descarga fluvial, por lo tanto el área de drenaje aumenta (Figura 1), y producen una rápida degradación o incisión de los materiales del lecho.

La potencia fluvial es variable entre las barrancas del volcán Popocatépetl (Figura 5). En general la potencia fluvial se incrementa con la distancia desde la divisoria (ríos 1, 2, 3, 4, 5, 6, 7, 11, y 12; Figura 5) con excepción de los ríos localizados en el flanco nororiental los cuales tienen sus valores máximos en un solo punto del río (ríos 8 , 9 y 10; Figura 5). El incremento de la potencia fluvial con la distancia en los ríos 1, 2, 3, 4, 5, 6, 7, 11, y 12 (Figura 5) es indicativo de la capacidad erosiva que tienen estos conforme aumenta la descarga fluvial. Es de resaltar el hecho que incluso en el río 1, (Barranca Nexpayantla, Tabla 1) los valores sean altos, no obstante que la incisión se produce sobre los materiales más antiguos del Popocatépetl (Figura 2) (Robin y Boudal, 1987; Espinasa-Pereña y Martín-Del Pozzo, 2006). En el caso de los ríos 8, 9 y 10 (Figura 5) los bajos valores en la potencia fluvial pueden estar relacionados con la edad y composición de los materiales, en el sector nororiental la incisión fluvial se produce sobre los flujos de lava del Holoceno, que corresponden con las unidades de lava más recientes del volcán (Espinasa-Pereña y Martín-Del Pozzo, 2006). Es posible que en este sector la erosión fluvial no haya podido formar valles profundos, sin embargo, los picos en la potencia fluvial (ríos 8, 9 y 10; Figura 4) sugieren que hay zonas en donde el incremento brusco de la pendiente del canal produce una mayor incisión (ver discusión sobre knickpoints más adelante).

La variabilidad que existe entre las barrancas del volcán Popocatépetl también está reflejada en el gráfico Chi (Figura 6). En los casos donde existe un equilibrio dinámico, la relación en el gráfico $C h i$ está dada por una linealidad en el perfil, cuando existe un incremento en las tasas de levantamiento tectónico la pendiente de la línea en el gráfico Chi aumenta (Perron y Royden, 2013). En la gran mayoría de los ríos del Popocatépetl la linealidad en los perfiles está interrumpida, además de que tienen una verticalidad variable (Figura 6). Los cambios en la verticalidad de los perfiles del gráfico Chi son indicativos del control que ejerce la litología frente a los procesos erosivos, en los cuales el sistema fluvial presenta distintos ajustes (Hack, 1973). La presencia de los knickpoints también es un factor que afecta la linealidad de los perfiles (Perron y Royden, 2013), en el caso del Popocatépetl estos fueron identificados en la mayoría de las barrancas (Tabla 2). En el gráfico Chi los ríos 5 y 12 presentan una notoria desviación con respecto a los demás perfiles (Figura 6), el valor $m / n$ de estos de $\sim 0.20$ y ambos tienen una topografía similar (Figura 3). Los valores $m / n$ son equivalentes a la concavidad $(\theta)$ determinada mediante la Ecuación $1 \mathrm{y}$, por lo tanto, guardan una cierta correlación con la verticalidad de los perfiles en coordenadas $\chi$. En el presente trabajo se detectó que la integral hipsométrica tiene una baja correlación, aunque significativa $(\mathrm{p}<0.05)$, con el valor $m / n$, tal situación denota que a menor concavidad, existe un mayor volumen de roca presente en la cuenca (Figura 7). En los ríos 5 y 12 la baja concavidad puede asociarse con la presencia de los flujos de lava de $\sim 14000$ y los depósitos piroclásticos $>900$ años respectivamente. Los ríos 1 y 7 presentan los valores $m / n$ más altos y los más bajos valores en la integral hipsométrica (Figura 7), tal condición puede también apreciarse en su perfil topográfico (Figura 3) en los cuales se advierte una morfología cóncava bien desarrollada.

En las barrancas del volcán Popocatépetl se detectaron numerosos knickpoints (Tabla 2; Figura 8) aunque sólo en algunos casos $(n=11)$ se pudo determinar el retroceso de los mismos (Tabla 2). La distancia del retroceso de los knickpoints guarda una moderada correlación con el área de drenaje (Figura 9A), lo que indica que la propagación de estas formas está controlada por la descarga fluvial, de tal forma que existe una relación proporcional. La relación entre el retroceso del knickpoint y el área de drenaje se ha confirmado en diversos sitios donde los knickpoints son propagados a consecuencia de una caída en el nivel de base (e.g., Bishop et al., 2005; Loget y van den Driessche, 2009; Castillo et al., 2013).

En el caso del volcán Popocatépetl, los distintos flujos de lava provocan cambios bruscos en las pendientes del terreno, sobre todo en los frentes de lava, en los cuales el sistema erosivo fluvial los registra como cambios en el nivel de base local, lo que produce la propagación aguas arriba de un knickpoint. Con las edades de las unidades morfoestratigráficas propuestas por Espinasa-Pereña y Martín-Del Pozzo (2006) se pudieron evaluar las tasas de propagación de los knickpoints (Tabla 2; Figura 9), la tasa media es de $0.05 \pm 0.02 \mathrm{~m} \mathrm{a}^{-1}$. Los valores más altos fueron de $\sim 0.17 \mathrm{~m} \mathrm{a}^{-1}$ (Figura 9B). A pesar de que las tasas de propagación son una estimación preliminar, debido a la incertidumbre que existe en las edades de las lavas, los resultados obtenidos (Figura 8) indican que los flujos de lava y en particular los frentes, afectan al sistema erosivo 

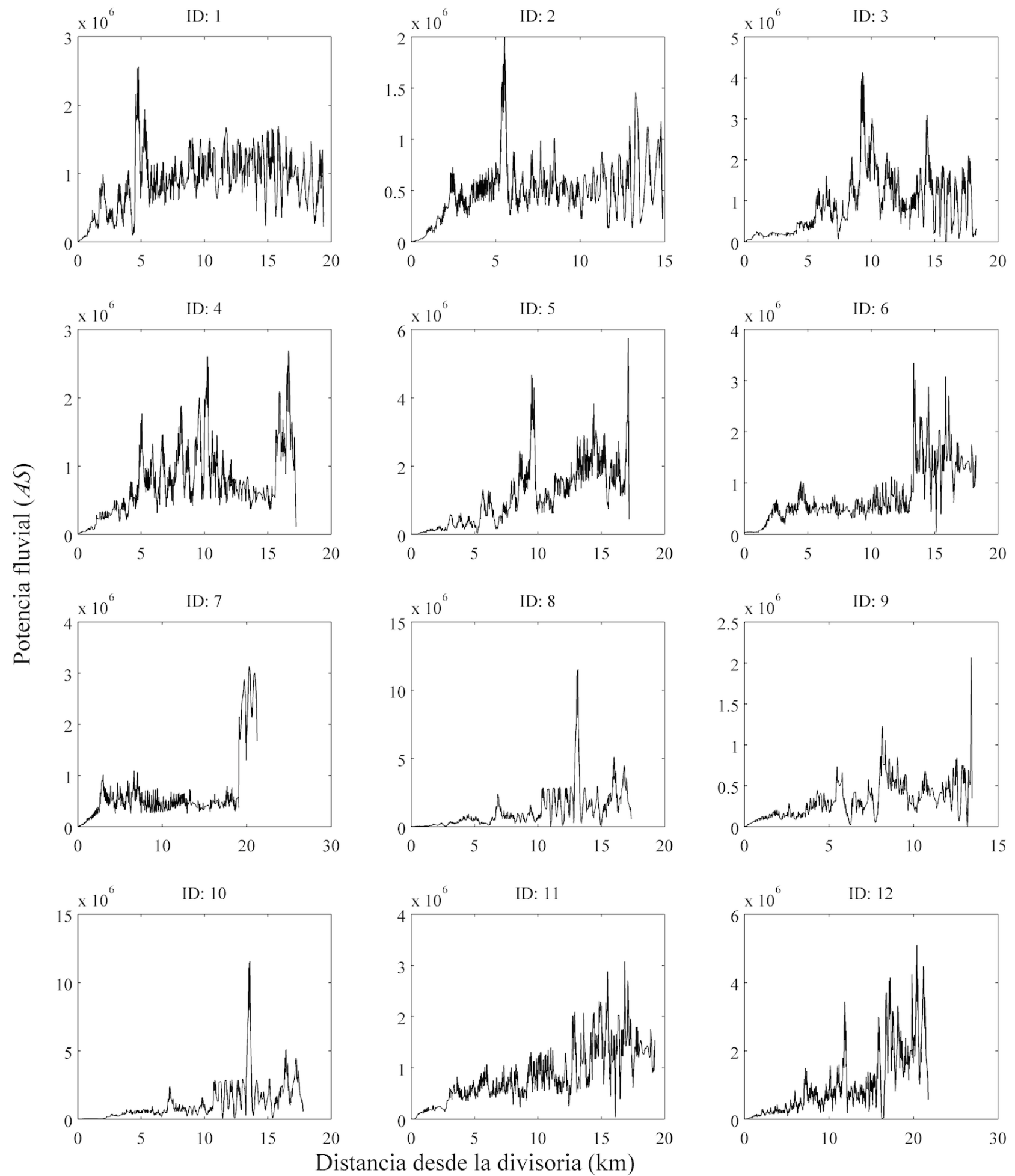

Figura 5. Gráficos de la potencia fluvial de las barrancas del volcán Popocatépetl. En los ríos 1, 2, 3, 4, 6, 7, 11 y 12 la potencia fluvial se incrementa aguas abajo. Los ríos 8, 9 y 10 presentan un incremento de la potencia fluvial donde existe un knickpoint (ver Figura 1 y Figura 2). 


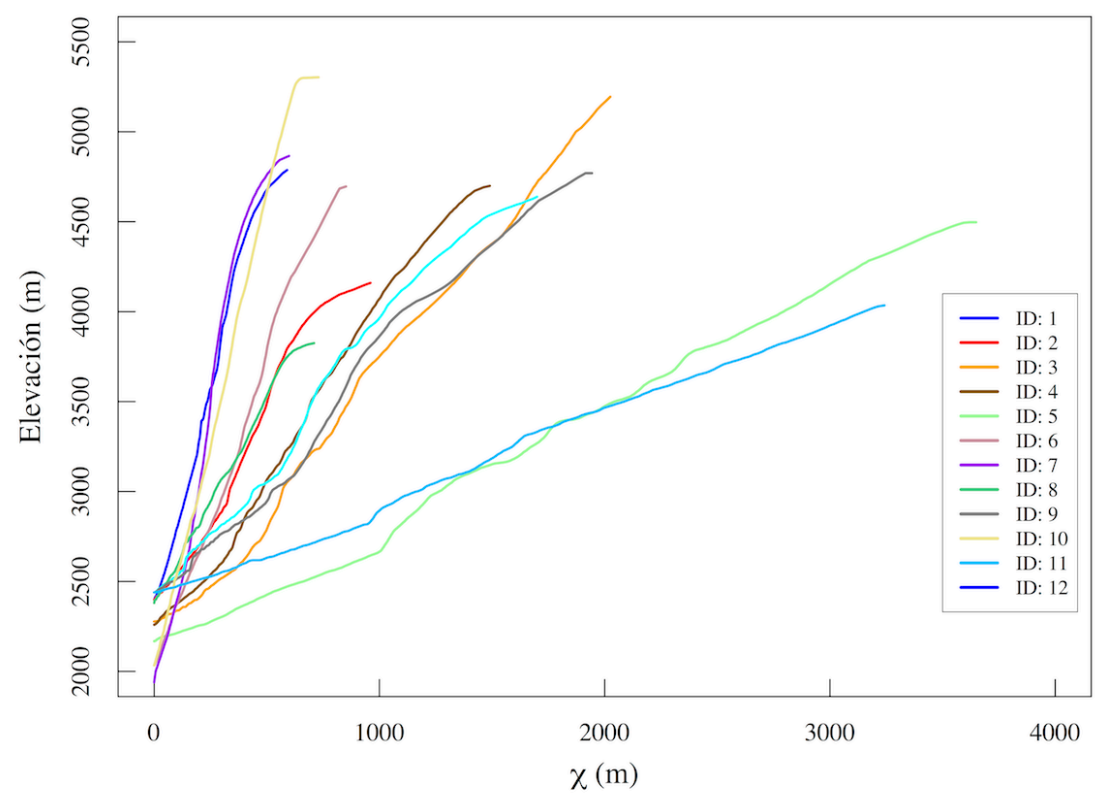

Figura 6. Gráfico Chi de las barrancas del Popocatépetl. Todas las barrancas tienen una discontinuidad de la linealidad y presentan variabilidad de su verticalidad. El ID corresponde con el número de río analizado (ver Tabla 1). Las barrancas 5 y 12 tienen una menor pendiente y en consecuencia un menor valor de $m / n$ (Tabla 1). Nótese que en las barrancas 5 y 12 el comportamiento de la potencia fluvial es similar (Figura 4).

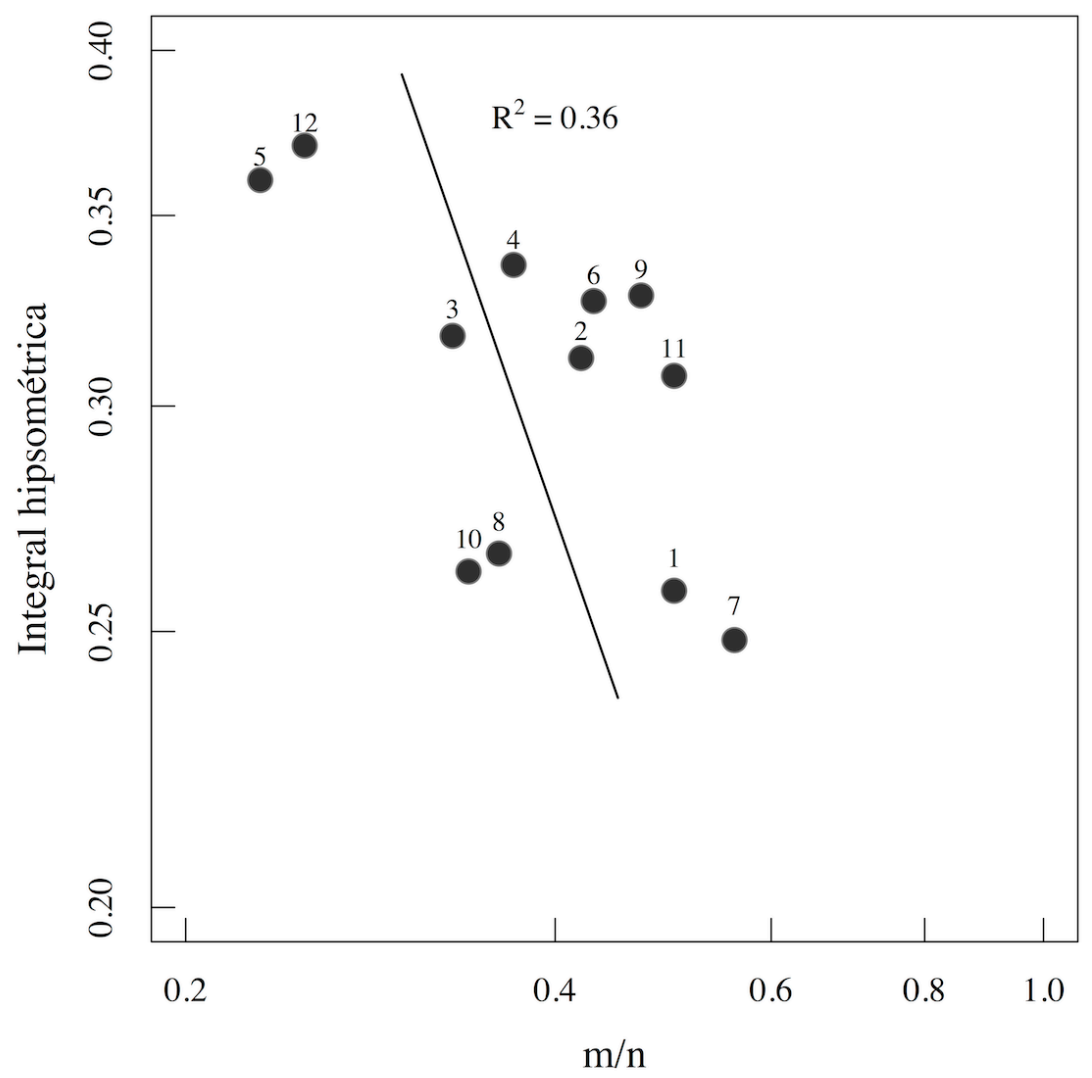

Figura 7. Correlación entre la integral hipsométrica y el valor $m / n$. El mejor ajuste de la curva es con una función potencial, la correlación es significativa $(\mathrm{p}<0.05)$. El modelo confirma la proporción inversa entre la concavidad y el volumen relativo de roca disponible en cada cuenca. 


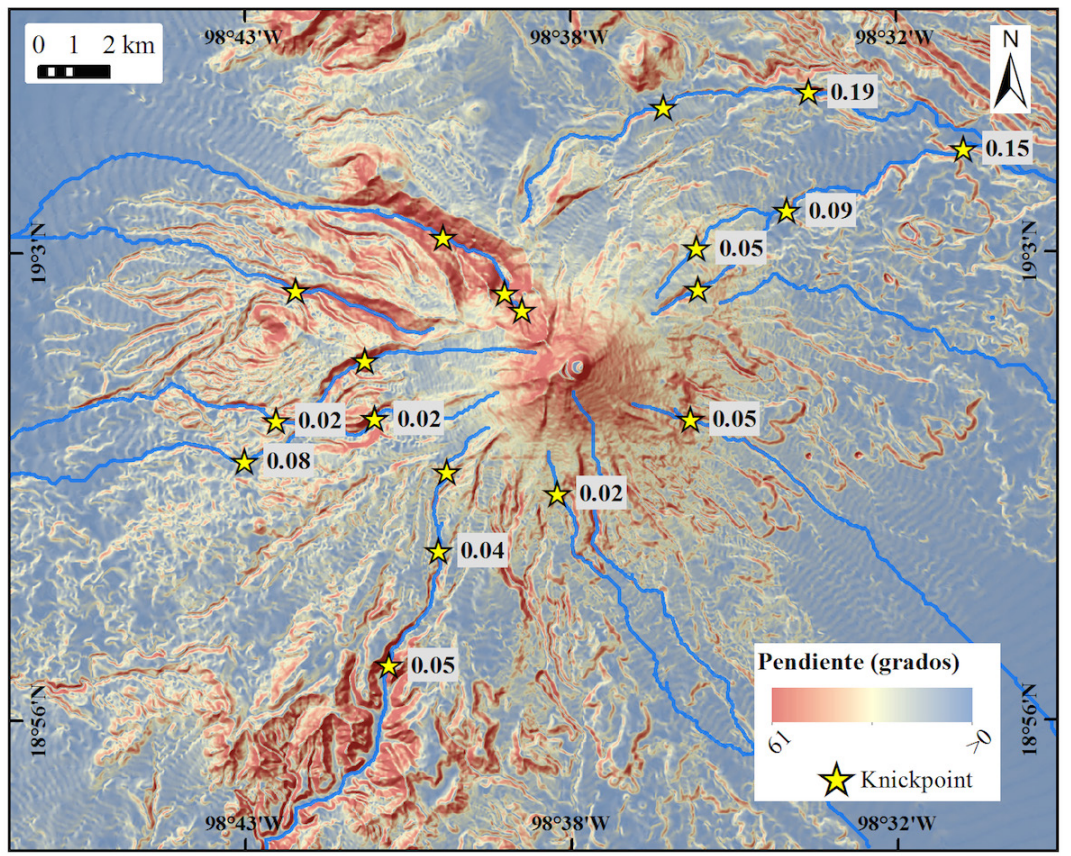

Figura 8. Mapa de las pendientes del terreno y localización de principales knickpoints. Los más altos valores de pendientes corresponden con las zonas de mayor relieve local. Nótese que los knickpoints del flanco nororiental, oriental, suroriental y sur están cerca del límite de los flujos de lava. Los números indican las tasas de retroceso $\left(\mathrm{m} \mathrm{a}^{-1}\right)$ de los knickpoints (Tabla 2).
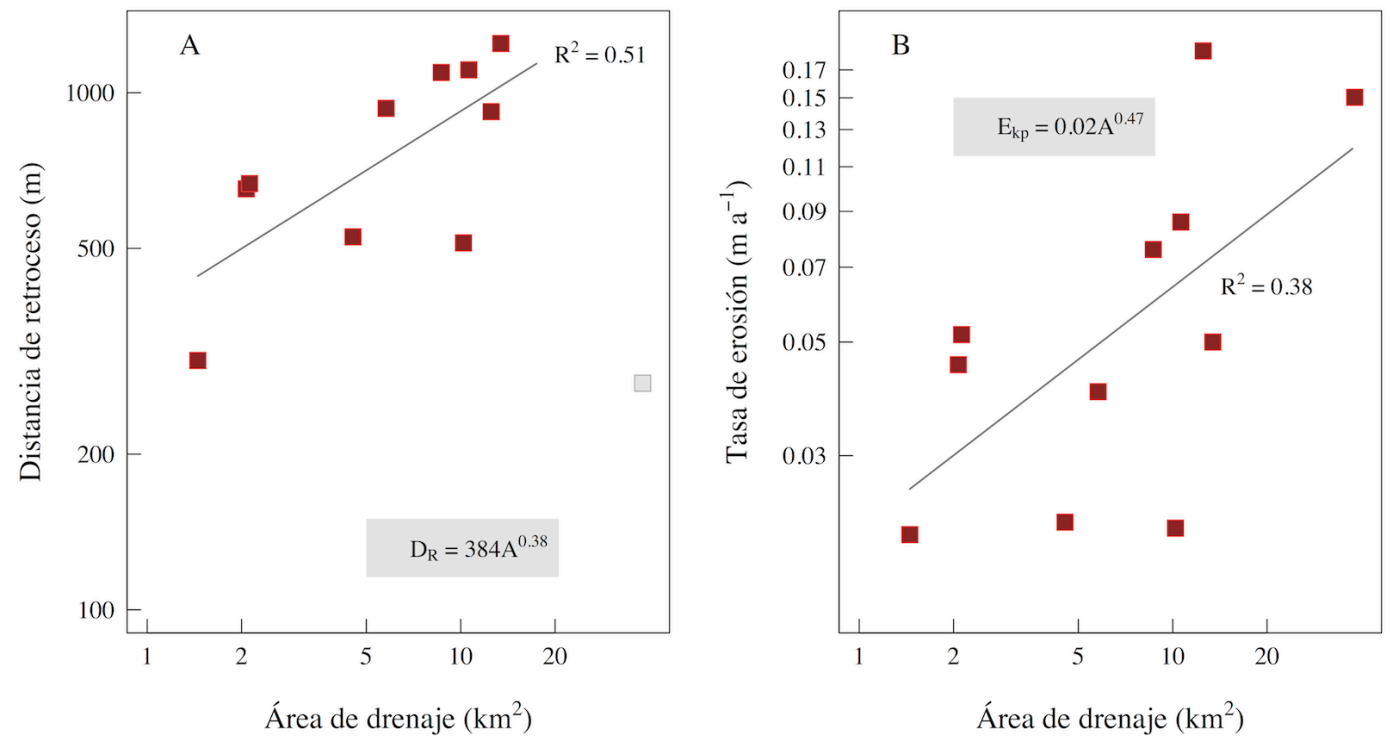

Figura 9. Gráficos de la distancia de retroceso y tasa de erosión versus el área de drenaje. Las correlaciones en A y B son significativas (p $<0.05$ ). Los gráficos en A y B revelan que la descarga fluvial controla el retroceso de los knickpoints. Nótese que los exponentes indican que incluso en cuencas pequeñas, los procesos fluviales pueden responder a perturbaciones en los lechos.

fluvial, lo que resulta en la migración aguas arriba de pulsos incisivos.

Si bien el grado de erosión del relieve volcánico es función de la edad de los materiales sobre los que inciden los ríos, la relación observada entre las tasas de retroceso de los knickpoints con el área de drenaje (Figura 9B) confirman que la descarga fluvial es un factor de primer orden en la erosión de los materiales volcánicos. La baja correlación observada entre las tasas de retroceso en el volcán Popocatépetl (Figura 9B) se debe en gran medida a la incertidumbre que se tiene en edades de las lavas y la generalización de las mismas, ya que la relación entre las tasas de retroceso de los knickpoints y el área de drenaje guardan una estrecha correlación independientemente de la edad (ver Figura 5 y 
Tabla 2 en Loget y van den Driesseche, 2009).

Las tasas de propagación estimadas son un orden más altas de aquellas reportadas por Franco (2009), sin embargo, este autor evaluó el caso de la incisión vertical cercana a las cabeceras, por lo que las tasas de erosión son menores al caso de la propagación de un knickpoint, el cual ocurre como un evento instantáneo (Gardner, 1983; Jansen et al., 2011). A pesar de que las tasas de retroceso están generalizadas y que no fue posible establecer una curva de retroceso de varios knickpoints sobre el material de una sola edad, nuestros datos sugieren que las tasas de retroceso en los materiales más antiguos son menores con respecto a los más jóvenes (Tabla 2). Debido a que en el retroceso de los knickpoints la pendiente del canal es un factor importante (Howard, 1998), la presencia de altos valores de pendiente a lo largo de las barrancas del volcán (Figura 4), sugiere que hay zonas donde puede haber altas tasas de erosión como también lo sugieren los valores de la potencia fluvial (Figura 5).

Los resultados del análisis morfométrico realizado en las cuencas indican que la media de los valores $k_{\mathrm{sn}}$ guarda una débil correlación con las pendientes del terreno (Figura 10A). La tendencia observada en la Figura 10A es concordante con los resultados obtenidos en otros ambientes donde los valores medios $k_{\mathrm{sn}}$ están escalados con las tasas de erosión (e.g., Ouimet et al., 2009; DiBiase et al., 2010; Cyr et al., 2010). Es posible que en el caso del Popocatépetl los valores $k_{\mathrm{sn}}$ no se correlacionen de forma más estrecha con las pendientes del terreno o su equivalente, el relieve local, porque en el caso del relieve volcánico los flujos de lava y el material piroclástico que cubre el relieve, genera que el sistema fluvial se forme por los límites entre los flujos de lava o los puntos más deprimidos del relieve sepultado, donde comienza el desarrollo de los valles, por lo tanto, en este tipo de relieve la relación que se observa entre incisión y evolución de las laderas (e.g., Reinhardt et al., 2007; Roering et al., 2007) puede no ocurrir. En zonas montañosas controladas por la tectónica, la respuesta incisiva está relacionada con la evolución de las laderas ya que a mayor incisión, mayor es la desestabilización de las laderas en la base y por lo tanto se desarrolla una morfología recta, donde los deslizamientos son continuos (Roering et al., 2007). La inconexión entre la morfología de las laderas y la incisión está reflejada en la diferencia que tiene el exponente de la correlación de las superficies cóncavas y convexas versus la pendiente media de la cuenca (ANCOVA, $\mathrm{p}<0.05$ ) (Figura 10B). La concavidad en este caso aumenta de forma más rápida conforme se incrementa la pendiente, mientras que la convexidad cambia de forma más lenta. Los análisis morfométricos de las cuencas y de los perfiles longitudinales de los ríos indican que en el volcán Popocatépetl existe una alta dinámica erosiva fluvial, que se incrementa con la pendiente local y ésta puede ser alta, incluso en áreas lejanas a las cabeceras. La historia eruptiva del Popocatépetl, junto con el aporte de un volumen importante de los productos volcánicos recientes, favorecen el estado de transitoriedad del relieve con respecto a los procesos erosivos fluviales. El Popocatépetl está por lo tanto, en un estado de desequilibrio donde no existe una relación entre la incisión y el desarrollo
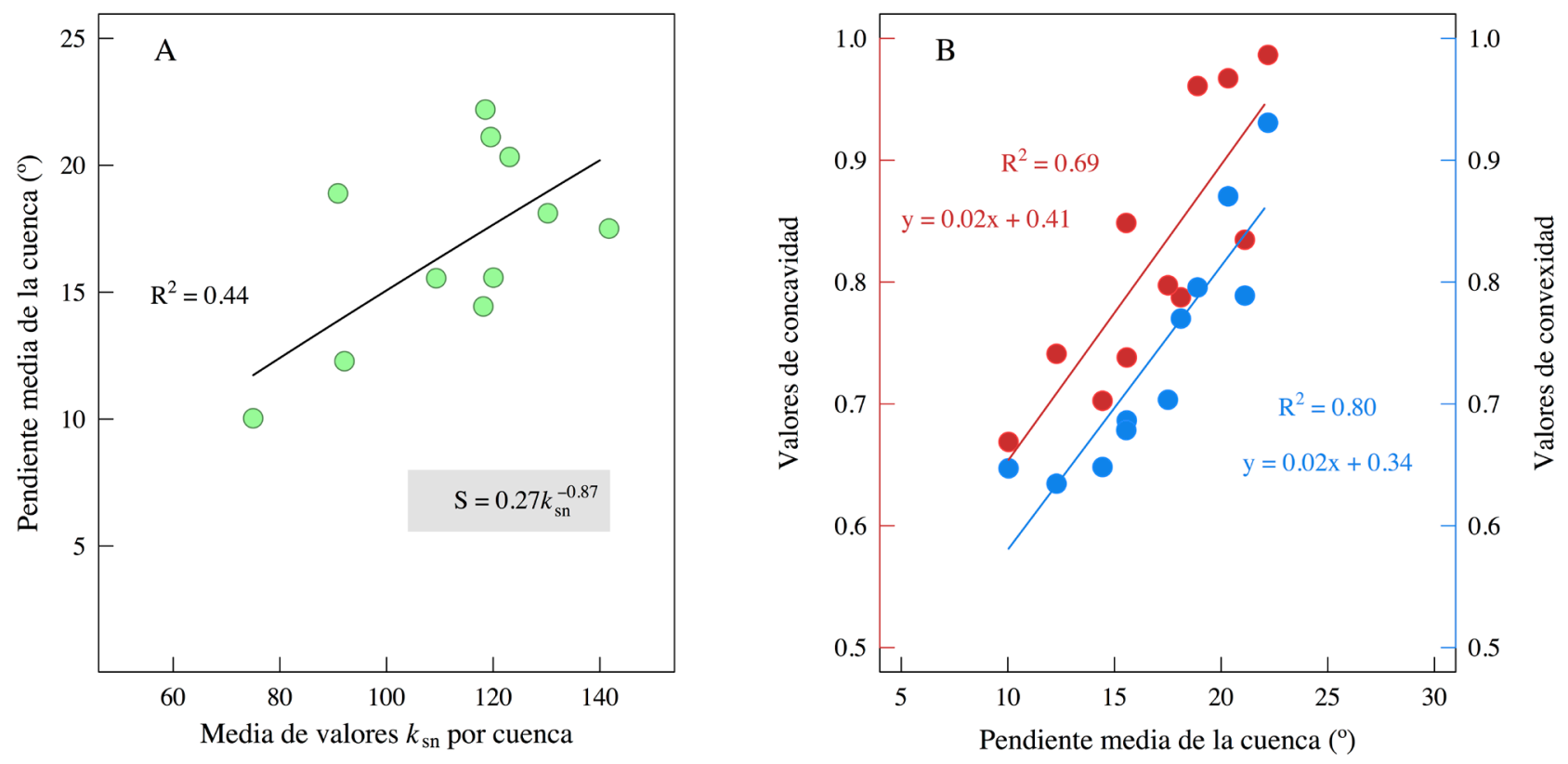

Figura 10. Relación entre la pendiente media de la cuenca y el valor medio $k_{\mathrm{sn}}$ de las cuencas (A) y correlación entre la pendiente media con la concavidad (B, eje vertical izquierdo) y convexidad (B, eje vertical derecho) media de la cuenca. La tendencia observada en A indica que una parte del relieve local (desnivel) se asocia a procesos erosivos fluviales. En B existen una diferencia significativa en la pendiente de las gráficas para convexidad y concavidad (test ANCOVA), condición que sugiere que no hay una relación directa entre la morfología de las laderas y la incisión y debido a esta última existe un incremento más rápido de la concavidad junto con el incremento de la pendiente o el desnivel del terreno. 
evolutivo de las laderas.

\section{Conclusiones}

Los resultados de esta investigación indican que debido a la intensa actividad volcánica en la historia reciente del volcán Popocatépetl ( $<23000$ años), el sistema erosivo fluvial se encuentra en estado de desequilibrio. Los flujos de lava producen un efecto de caída de nivel de base local, el cual desencadena la propagación de knickpoints a tasas de $\sim 0.05 \mathrm{~m} \mathrm{a}^{-1}$. En el volcán no se observa un límite claro entre el dominio de los procesos de ladera, como son los flujos de derrubios, y el dominio controlado por la dinámica fluvial. El análisis de la relación entre el gradiente de los canales y el área de descarga indican que los flujos de derrubios pueden presentarse más allá del área crítica de $10^{5} \mathrm{~m}^{2}$. Los datos obtenidos en esta investigación indican que las barrancas del volcán Popocatépetl tienen una alta capacidad erosiva la cual aumenta en muchos de los casos hacia las partes bajas del edificio volcánico, tal hecho presenta una condición de amenaza por procesos fluviales o flujos de derrubios a las poblaciones localizadas en los abanicos formados en las desembocaduras de las barrancas del volcán.

\section{Agradecimientos}

Los autores del manuscrito agradecen al Dr. José Luis Macías y a tres revisores anónimos sus comentarios y críticas constructivas que mejoraron la versión final del presente trabajo.

\section{Referencias}

Ahnert, F., 1970, Functional relationships between denudation, relief, and uplift in large mid-latitude drainage basins: American Journal of Science, 268, 243-263.

Anderson, R.S., Molnar, P., Kessler, M.A., 2006, Features of glacial valley profiles simply explained: Journal of Geophysical Research, 111, F01004.

Attal, M., Tucker, G., Whittaker, A.C., Cowie, P., Roberts, G., 2008, Modeling fluvial incision and transient landscape evolution: influence of dynamic channel adjustment: Journal of Geophysical Research, 113, F02005.

Attal, M., Cowie, P.A., Whittaker, A.C., Hobley, D., Tucker, G.E., Roberts, G.P., 2011, Testing fluvial erosion models using the transeint response of bedrock rivers to tectonic forcing in the Apennines, Italy: Journal of Geophysical Research, 116, F02005.

Binnie, S.A., Phillips, W.M., Summerfield, M.A., Fifield, L.K., 2007, Tectonic uplift, threshold hillslopes, and denudation rates in a developing mountain range: Geology, 35, 743-746.

Bishop, P., 2007, Long-term landscape evolution: linking tectonics and surface processes: Earth Surface Processes and Landforms, 32, 329-365.

Bishop, P., Hoey, T., Jansen, J., Lexartza, I., 2005, Knickpoint recession rate and catchment area: the case of uplifted rivers in Eastern Scotland: Earth Surface Processes and Landforms, 30, 767-778.
Brocklehurst, S., Whipple, K., 2006, Assessing the relative efficiency of fluvial and glacial erosion through simulation of fluvial landscapes: Geomorphology, 75, 283-299.

Brozović, N., Burbank, D.W., Meigs, A.J., 1997, Climatic limits on landscape development in Northwestern Himalaya: Nature, 276, 571-574.

Capra, L., Poblete, M.A., Alvarado, R., 2004, The 1997 and 2001 lahars of Popocatépetl volcano (Central Mexico): textural and sedimentological constrains on their origin and hazards: Journal of Volcanology and Geothermal Research, 131, 351-369.

Castillo, M., Lugo-Hubp, J., 2011, Estado actual del conocimiento, clasificación y propuesta de inclusión del término knickpoint en el léxico geológico-geomorfológico del español: Boletín de la Sociedad Geológica Mexicana, 63, 353-364.

Castillo, M., Bishop, P., Jansen, J.D., 2013, Knickpoint retreat and transient bedrock channel morphology triggered by base-level fall in small bedrock river catchments: the case of the Isle of Jura, Scotland: Geomorphology, 180-181, 1-9.

Church, M., Ryder, J.M., 1972, Paraglacial sedimentation: A consideration of fluvial processes conditioned by glaciation: Geological Society of America Bulletin, 83, 3059-3075.

Conte, G., Urrutia-Fucugauchi, J., Soler-Arechalde, A.M., Morton-Bermea, O., 2004, Paleomagnetic study of the lavas from the Popocatepetl volcanic region, Central Mexico: International Geology Review, $46,210-225$.

Cyr, A., Granger, D., Olivetti, V., Molin, P., 2010, Quantifying rock uplift rates using channel steepness and cosmogenic nuclidedetermined erosion rates: Examples from northern and southern Italy: Lithosphere, 2, 188-198.

Delgado-Granados, H., 1997, The glaciers of Popocatépetl volcano (Mexico): Changes and causes: Quaternary International, 43-44, 53-60.

DiBiase, R., Whipple, K., Heimsath, A., Ouimet, W., 2010, Landscape form and millenial erosion rates in the San Gabriel Mountains, CA: Earth and Planetary Science Letters, 289, 134-144.

Duvall, A., Kirby, E., Burbank, D., 2004, Tectonic and lithologic controls on bedrock channel profiles and processes in coastal California: Journal of Geophysical Research, 109, F03002.

Espinasa-Pereña, R., Martín-Del Pozzo, A.L., 2006, Morphostratigraphic evolution of Popocatépetl volcano, México, en Siebe, C., Macías, J.L., Aguirre-Díaz, G. (eds.), Neogene-Quaternary continental margin volcanism: A perspective from Mexico, Geological Society of America Special Paper, 402: Boulder, Colorado, Geological Society of America, 101-123.

Fernández-Eguiarte, A., Zavala-Hidalgo, J., Romero-Centeno, R., 2014, Atlas Climático Digital de México: México, D.F., Centro de Ciencias de la Atmósfera, Universidad Nacional Autónoma de México. Publicado desde el 21 de Enero de 2009, disponible en $<$ http:// uniatmos.atmosfera.unam. $\mathrm{mx}>$

Finlayson, D., Montgomery, D., 2003, Modeling large-scale fluvial erosion in geographic information systems: Geomorphology, 53, 147-164.

Finnegan, N., Roe, G., Montgomery, D., Hallet, B., 2005, Controls on the channel width of rivers: Implications for modeling fluvial incision of bedrock: Geology, 33, 229-232.

Finnegan, N.J., Hallet, B., Montgomery, D.R., Zeitler, P.K., Stone, J.O., Anders, M.A., Yuping, L., 2008, Coupling of rock uplift and river incision in the Namche Barwa-Gyala Peri massif, Tibet: Geological Society of America Bulletin, 120, 142-155.

Franco, R.O., 2005, Geomorfología del volcán Popocatépetl: México, Ciudad Universitaria, Facultad de Filosofía y Letras, UNAM, tesis de licenciatura, $150 \mathrm{p}$.

Franco, R.O., 2009, Procesos morfodinámicos en la vertiente Norte del Volcán Popocatépetl: México, Ciudad Universitaria, Facultad de Filosofía y Letras, UNAM, tesis de maestría, 144 p.

Gardner, T., 1983, Experimental study of knickpoint and longitudinal profile evolution in cohesive, homogeneous material: Geological Society of America Bulletin, 94, 664-672. 
Garzón, G., Ortega, J.A., Garrote, J., 2008, Morfología de perfiles de ríos en roca. Control tectónico y significado evolutivo en el Bajo Guadiana: Geogaceta, 4, 63-66.

Hack, J.T., 1957, Studies of longitudinal stream profiles in Virginia and Maryland: USGS Professional Paper, 249, 3, 45-97.

Hack, J.T., 1973, Stream-profile analysis and stream gradient index: Journal of Research, 1, 421-429.

Harkins, N., Kirby, E., Heimsath, A., Robinson, R., Reiser, U., 2007, Transient fluvial incision in the headwaters of the Yellow River, northeastern Tibet, China: Journal of Geophysical Research, 112, F03S04.

Heine, K., 1994, The late-glacial moraine sequences in Mexico: is there evidence for the Younter Dryas event?: Palaeogeography, Palaeoclimatology, Palaeoecology, 112, 113-123.

Howard, A., 1994, A detachment-limited model of drainage basin evolution: Water Resources Research, 30, 7, 2261-2285.

Howard, A., 1998, Long profile development of bedrock channels: interactions of weathering, mass wasting, bed erosion and sediment transport, en Tinkler, K., Wohl, E. (eds.), Rivers Over Rock: Fluvial Processes in Bedrock Channels (Geophysical Monographs 107): Washington, D.C, American Geophysical Union, USA, 297-317.

Howard, A., Kerby, G., 1983, Channels changes in badlands: Geological Society of America Bulletin, 94, 6, 739-752.

Hurst, D., Mudd, S.M., Walcott, R., Attal, M., Yoo, K., 2012, Using hilltop curvature to derive the spatial distribution of erosion rates: Journal of Geophysical Research, 117, F02017.

Instituto Nacional de Estadística y Geografía (INEGI), 2012, Delimitación de Zonas Metropolitanas de México 2010: México, Instituto Nacional de Geografía Estadística y Geográfica, 216 p.

Jansen, J.D., Fabel, D., Bishop, P., Xu, S., Schnabel, C., Codilean, A.T., 2011, Does decreasing paraglacial sediment supply slow knickpoint retreat?: Geology, 39, 543-546.

Julio-Miranda, P., Delgado-Granados, H., 2003, Fast hazard evaluation employing digital photogrammetry: Popocatepetl glaciers, Mexico: Geofísica Internacional, 42, 2, 275-283.

Julio-Miranda, P., Delgado-Granados, H., Huggel, C., Kääb, A., 2005, Impact fo the eruptive activity on glacier evolution at Popocatépetl volcano (México) during 1994-2004: Journal of Volcanology and Geothermal Research, 170, 86-98.

Kirby, E., Whipple, K., 2001, Quantifying differential rock-uplift rates via stream profile analysis: Geology, 29, 5, 415-418.

Kirby, E., Whipple, K.X., 2012, Expression of active tectonics in erosional landscapes: Journal of Structural Geology, 44, 54-75.

Kirby, E., Whipple, K.X., Tang, W., Chen, Z., 2003, Distribution of active rock uplift along the eastern margin of the Tibetan Plateau: Inferences from bedrock channel longitudinal profiles: Journal of Geophysical Research, 108, B4.

Lachniet, M.S., Vazquez-Selem, L., 2005, Last Glacial Maximum equilibrium line altitudes in the circum-Carribean (Mexico, Guatemala, Costa Rica, Colombia, and Venezuela): Quaternary International, 138-139, 129-144.

Larue, J.P., 2008, Effects of tectonics and lithology on long profiles of 16 rivers of the southern Central Massif border between the Aude and the Orb (France): Geomorphology, 93, 343-367.

Loget, N., van den Driesseche, J., 2009, Wave train model for knickpoint migration: Geomorphology, 106, 376-382.

Macías, J.L., 2005, Geología e historia eruptiva de algunos de los grandes volcanes activos de México: Boletín de la Sociedad Geológica Mexicana, 52, 379-424.

Miller, J., 1991, The influence of bedrock geology on knickpoint development and channel-bed degradation along downcutting streams in south-central Indiana: Journal of Geology, 99, 591-605.

Montgomery, D., 2001, Slope distributions, threshold hillslopes and steady-state topography: American Journal of Science, 301, 432-454.

Montgomery, D.R. Brandon, M.T., 2002, Topographic controls on erosion rates in tectonically active mountain ranges. Earth and Planetary Science Letters, 202, 481-489.

Montgomery, D.R., Dietrich, W.E., 1988, Where do channels begin?: Nature, 336, 232-234.
Montgomery, D., Foufoula-Georgiou, E., 1993, Channel network source respresentation using digital elevation models: Water Resources Research, 29, 12, 3925-3934.

Montgomery, D., Gran, K., 2001, Downstream variations in the width of bedrock channels: Water Resources Research, 37, 6, 1841-1846.

Muñoz-Salinas, E., Manea, M.C., Palacios, D., Castillo, M., 2007, Estimation of lahar flow velocity on Popocatépetl volcano (Mexico): Geomorphology, 92, 91-99.

Muñoz-Salinas, E., Castillo, M., Manea, Manea, M., Palacios, D., 2010, On the geochronological method versus flow simulation software application for lahar risk mapping: A case study of Popocatépetl volcano, Mexico: Geografiska Annaler Series A: Physical Geography, 92, 311-328.

Norton, K.P., Abbühl, L.M., Schlunegger, F., 2010, Glacial conditioning as an erosional driving force in the Central Alps: Geology, 38, 655-658.

Ollier, C.D., 1988, Volcanoes: Oxford, Blackwell, 177 p.

Ouimet, W., Whipple, K., Granger, D., 2009, Beyond threshold hillslope: Channel adjustment to base-level fall in tectonically active mountain ranges: Geology, 37, 579-582.

Palacios, D., 1996, Recent geomorphologic evolution of a glaciovolcanic active stratovolcano: Popocatepetl (Mexico): Geomorphology, 16, 319-335.

Palacios, D., Zamorano, J.J., Gómez, A., 2001, The impact of present lahars on the geomorphologic evolution of proglacial gorges: Popocatepetl, Mexico: Geomorphology, 37, 15-42.

Perron, J.T., Royden, L., 2013, An integral approach to bedrock river profile analysis: Earth Surface Processes and Landforms, 38, 570-576.

Pike, R., Wilson, S., 1971, Elevation-Relief ration, hypsometric integral and geomorphic area-altitude analysis: Geological Society of America Bulletin, 82, 1079-1084.

Reinhardt, L., Bishop, P., Hoey, T.B., Dempster, T.J., Sanderson, D.C.W., 2007, Quantification of the transient response to base-level fall in a small mountain catchment: Sierra Nevada, southern Spain: Journal of Geophysical Research, 112, F03S05.

Robin, C., Boudal, C., 1987, A gigantic Bezymianny-Type event at the beginning of modern volcan Popocatépetl: Journal of Volcanology and Geothermal Research, 31, 115-129.

Roe, G., Montgomery, D., Hallet, B., 2002, Effects of orographic precipitation variations on the concavity of steady-state river profiles: Geology, 30, 2, 143-146.

Roering, J.J., Kirchner, J.W., Sklar, L.S., Dietrich, W.E., 2001, Hillslope evolution by nonlinear creep and landsliding: An experimental study: Geology, 29, 143-146

Roering, J.J., Perron, J.T., Kirchner, W.J., 2007, Functional relationships between denudation and hillslope form and relief: Earth and Planetary Science Letters, 264, 245-258.

Royden, L., Perron, T., 2013, Solutions of the stream power equation and application to the evolution of river longitudinal profiles: Journal of Geophisical Research: Earth Surface, 118, 1-22.

Safran, E.B., Bierman, P., Aalto, R., Dunne, T., Whipple, K.X., Caffe, M., 2005, Erosion rates driven by channel network incision in the Bolivian Andes: Earth Surface Processes and Landforms, 30, 1007-1024.

Schaaf, P., Stimac, J., Siebe, C., Macías, J.L., 2005, Geochemical evidence for mantle origin and crustal processes in volcanic rocks from Popocatépetl and surrounding monogenetic volcanoes, Central Mexico: Journal of Petrology, 46, 6, 1243-1282.

Schlunegger, F., Norton, K.P., Zeilinger, G., 2011, Climatic forcing on channel profiles in the Eastern Cordillera of the Coroico Region, Bolivia: Journal of Geology, 119, 97-107.

Schoenbohm, L., Whipple, K., Burchfiel, B., 2004, Geomorphic constrains on surface uplift, exhumation and plateau growth in the Red River region, Yunnan Province, China: Geological Society of America Bulletin, 116, 895-909.

Seidl, M., Dietrich, a., Kirchner, W., 1994, Longitudinal profile development into bedrock: An analysis of Hawaiian channels: Journal of Geology, 102, 457-474.

Siebe, C., Abrams, M., Macías, J.L., 1995, Volcán Popocatépetl: Estudios realizados durante la crisis de 1994-995: Centro Nacional de 
Prevención de Desastres, México, 195-220.

Sklar, L., Dietrich, W., 1998, River longitudinal profiles and bedrock incision models: stream power and the influence of sediment supply, en Tinkler, K., Wohl, E. (eds.), Rivers Over Rock: Fluvial Processes in Bedrock Channels: Washington, D.C., American Geophysical Union, 237-260.

Snyder, N., Whipple, K., Tucker, G., Merrits, D., 2003, Channel response to tectonic forcing: Field analysis of stream morphology in the Mendocino triple junction region, northern California: Geomorphology, 53, 97-127.

Stock, J., Montgomery, D., 1999, Geologic constrains on bedrock river incision using the stream power law: Journal of Geophysical Research, 104, B3, 4983-4993.

Stolar, D., Willet, S., Montgomery, D., 2007, Characterization of topographic steady state in Taiwan: Earth and Planetary Science Letters, 261, 421-431.

Strahler, A., 1952, Hypsometric (area-altitude) analysis of erosional topography: Bulletin of the Geological Society of America, 63, 1117-1142.

Tarboton, D., Bras, R., Rodriguez-Iturbe, I., 1991, On the extraction on channel networks from digital elevation data: Hydrological Processes, 5, 81-100.

Thouret, J.C., 1999, Volcanic geomorphology - an overview: Earth-Science Reviews, 47, 95-131.

Trauerstein, M., Norton, K.P., Preusser, F., Schlunegger, F., 2013, Climatic imprint on landscape morphology in the western escarpment of the Andes: Geomorphology, 194, 76-83.

Turowski, J., Hovius, N., Meng-Long, H., Lague, D., Men-Chiang, C., 2008, Distribution of erosion across bedrock channels: Earth Surface Processes and Landforms, 33, 353-363.

Vázquez-Selem, L., Heine, K., 2011, Late Quaternary Glaciation in Mexico, en Ehler, J., Gibbard, P.L., Hughes, P.D. (eds.), Quarternary Glaciations - Extent and Chronology: A Closer Look: Amsterdam, Elsevier, 849-861.

Whipple, K., 2004, Bedrock rivers and the geomorphology of active orogens: Annual Review Earth Planetary Sciences, 32, 151-185.

Whipple, K., Tucker, G., 1999, Dynamics of the stream-powr model: Implications for the height limits of mountain ranges, landscape response timescales and research needs: Journal of Geophysical Research, 104, B8, 17661-17674.

Whipple, K., Kirby, E., Brocklehurst, S., 1999, Geomorphic limits to climate-induced increases in topographic relief: Nature, 401, 39-43.

Whipple, K., Snyder, N., Dollenmayer, K., 2000, Rates and processes of bedrock incision by the Upper Ukak River since the 1912 Novarupta ash flow in the Valley of Ten Thousand Smokes: Geology, 28, 9, 835-838.

White, S.E., 1981, Neoglacial to recent glacier fluctuations on the volcano Popocatépetl, Mexico: Journal of Glaciology, 27, 96, 359-363.

White, S.E., 2002, Glaciers of North America - Glaciers of México, en Williams, R.S., Ferrigno, J.G. (eds.), Satellite Image Atlas of Glaciers of the World: U.S., Geological Survey, 383-404.

Whittaker, A.C., Attal, M., Cowie, P.A., Tucker, G.E., Roberts, G., 2008, Decoding temporal and spatial patterns of fault uplift using transient river long profiles: Geomorphology, 100, 506-526.

Willgoose, G., 1994, A physical explanation for an observed area-slopeelevation relationship for catchments with declinig relief: Water Resources Research, 30, 2, 151-159.

Willgoose, G., Bras, R., Rodriguez-Iturbe, I., 1994, Hydrogemorphology modelling with a physically based river basin evolution model, en Kirkby, M. (ed.), Process Models and Theoretical Geomorphology: USA, John Wiley and Sons, 271-294.

Wobus, C., Crosby, B., Whipple, K., 2006, Hanging valleys in fluvial systems: Controls on the occurrence and implications for landscape evolution: Journal of Geophysical Research, 111, F02017.

Wohl, E., 2004, Limits to downstream hydraulic geometry: Geology, 35, 897-900.

Ye, F.Y., Barriot, J., Carretier, S., 2013, Inititation and recession of the fluvial knickpoints of the Island of Tahiti (French Polynesia): Geomorphology, 186, 162-173.

Manuscrito recibido: Febrero 25, 2014

Manuscrito corregido recibido: Septiembre 2, 2014

Manuscrito aceptado: Noviembre 11, 2014 